\title{
Optimizing Dyadic Nets
}

\author{
ABDALLA G. M. AHMED, KAUST, KSA \\ PETER WONKA, KAUST, KSA
}

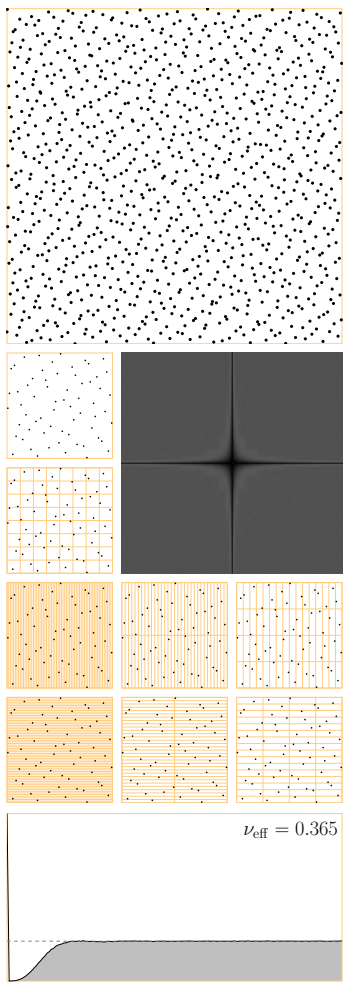

(a) Netshuffle, using random flags

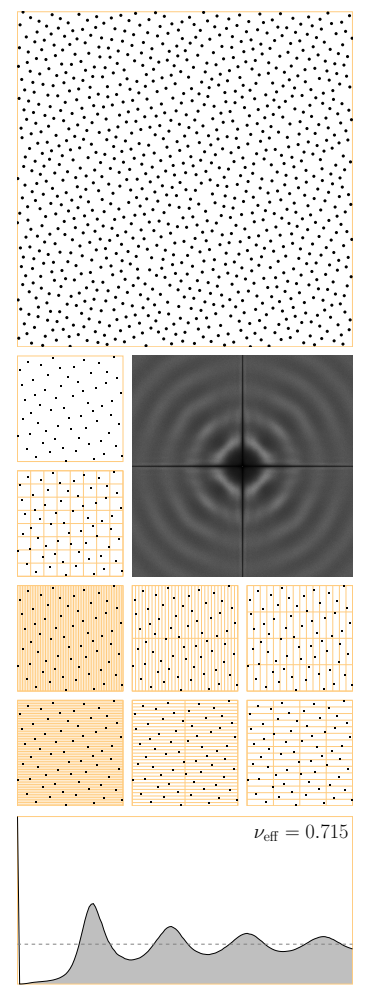

(b) Poisson Disk, average $r_{\mathrm{f}}=0.74$

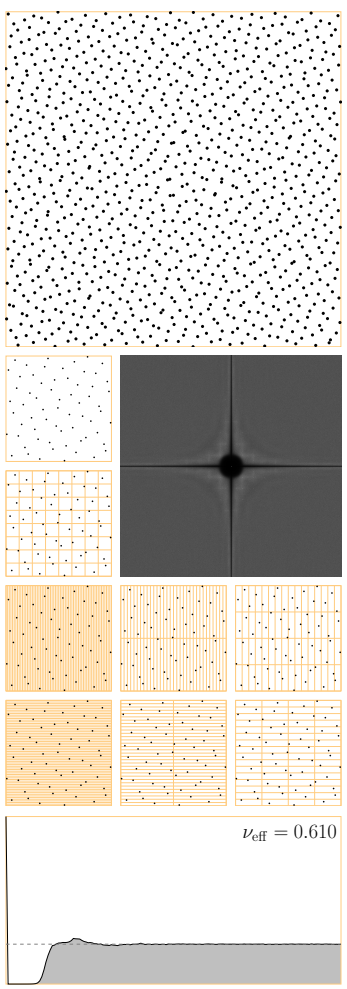

(c) Blue Noise, $\sigma=1.0$

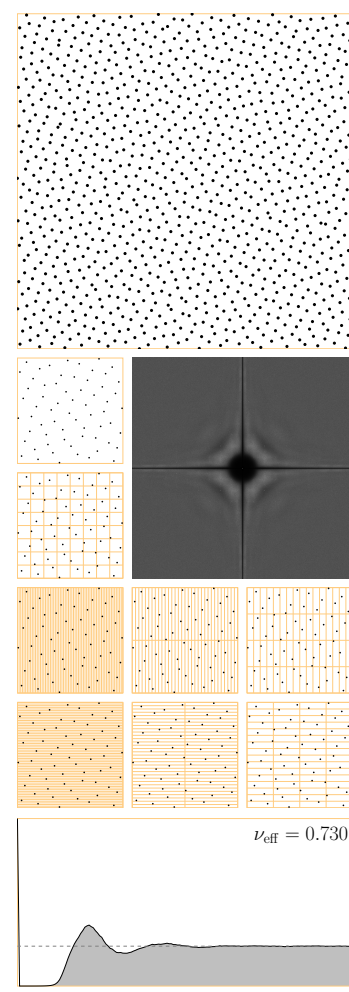

(d) Blue Noise, $\sigma=0.75$

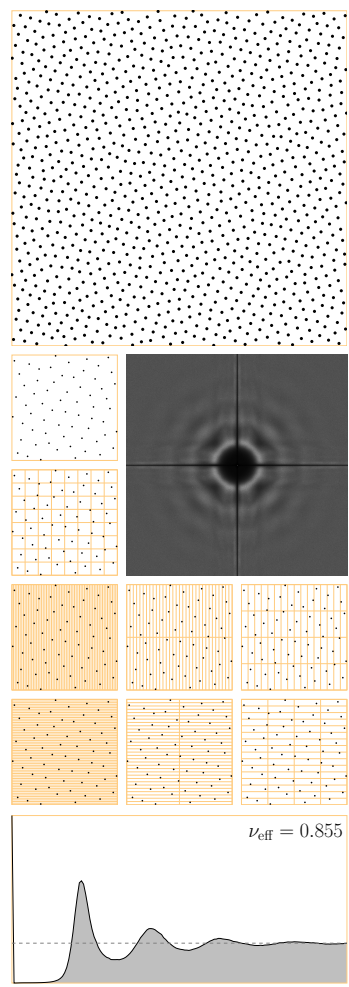

(e) Blue Noise, $\sigma=0.5$

Fig. 1. Various $(0, m, 2)$-nets with different spectral profiles produced by our (a) Netshuffle and (b-e) Netoptimize algorithms. In each of the five columns, the top plot shows a 1024-point set. The middle-right image shows the corresponding frequency power spectrum, and the bottom plot shows its radial average, both obtained by averaging 100 periodograms of 4096-point sets, using the standard PSA tool [Schlömer and Deussen 2011] with the default settings. The eight subplots show a 64-point net of the same profile, demonstrating the dyadic net property that the points are stratified over all the elementary intervals. The set in the top subplot is similarly stratified over all 11 possible stratifications. The Netshuffle version in (a) is close to Owen's scrambling, but is free of the grid-like artifacts typically seen in its power spectrum. The blue-noise profiles are obtained by adjusting the $\sigma$ parameter of a Gaussian optimization process.

We explore the space of $(0, m, 2)$-nets in base 2 commonly used for sampling We present a novel constructive algorithm that can exhaustively generate all nets - up to $m$-bit resolution - and thereby compute the exact number of distinct nets. We observe that the construction algorithm holds the key to defining a transformation operation that lets us transform one valid net into another one. This enables the optimization of digital nets using arbitrary

Authors' addresses: Abdalla G. M. Ahmed, KAUST, KSA, abdalla_gafar@hotmail.com Peter Wonka, KAUST, KSA, pwonka@gmail.com.

Permission to make digital or hard copies of all or part of this work for personal or classroom use is granted without fee provided that copies are not made or distributed for profit or commercial advantage and that copies bear this notice and the full citation on the first page. Copyrights for components of this work owned by others than the author(s) must be honored. Abstracting with credit is permitted. To copy otherwise, or republish, to post on servers or to redistribute to lists, requires prior specific permission and/or a fee. Request permissions from permissions@acm.org.

(C) 2021 Copyright held by the owner/author(s). Publication rights licensed to ACM. 0730-0301/2021/8-ART141 \$15.00

https://doi.org/10.1145/3450626.3459880 objective functions. For example, we define an analytic energy function for blue noise, and use it to generate nets with high-quality blue-noise frequency power spectra. We also show that the space of $(0,2)$-sequences is significantly smaller than nets with the same number of points, which drastically limits the optimizability of sequences.

\section{CCS Concepts: • Computing methodologies $\rightarrow$ Rendering}

Additional Key Words and Phrases: sampling, nets, digital nets, dyadic nets, Sobol sequences, quasi-Monte Carlo, blue noise, low-discrepancy sequences, Owen's scrambling, Faure-Tezuka scrambling

\section{ACM Reference Format:}

Abdalla G. M. Ahmed and Peter Wonka. 2021. Optimizing Dyadic Nets. ACM Trans. Graph. 40, 4, Article 141 (August 2021), 17 pages. https://doi.org/10. $1145 / 3450626.3459880$ 


\section{INTRODUCTION}

Sample generation is ubiquitous in computer graphics and used extensively in halftoning, stippling, geometry processing, machine learning, and Monte Carlo-based rendering. In computer graphics, we can identify two interesting lines of work in the uniform distribution of samples: (A) blue-noise sampling and (B) low-discrepancy (LD) sets and sequences.

Blue-noise [Ulichney 1987, 1988], or Poisson-disk [Dippé and Wold 1985], sampling generates visually pleasing patterns in 2D. The main advantages of blue-noise sampling are that the point distribution has a low-energy, low-frequency band in its frequency power spectrum and a large Poisson-disk radius, which refers to the minimal spacing between the points. Both features are desirable for many applications [Durand 2011; Öztireli 2016; Ulichney 1987, 1988]. Based on insights from the classic sampling theory [Shannon 1948], the patterns are generally considered the best for many 2D applications, including halftoning, stippling, and reconstruction. Following a technical report by Durand [2011], there is also growing interest in using blue-noise sampling for Monte Carlo integration [Öztireli 2016; Pilleboue et al. 2015; Ramamoorthi et al. 2012; Subr and Kautz 2013]. This, however, remains only a theoretical interest, as no end-to-end industrial-quality rendering samplers have been presented.

As an alternative to blue noise, Shirley [1991] and Mitchell [1992] introduced LD sampling patterns in computer graphics. Of special interest are so-called nets, mainly Sobol sequences, that are well-suited to processing by computers. Indeed, the cost of generating Sobol sequences is comparable to that of pseudo-random numbers. One downside is that these patterns are more structured than blue noise, with their energy concentrated at scattered frequency spikes. While this may lead to some aliasing artifacts, the very low computational cost of LD sequences like Sobol's and Halton's, their impressive convergence rates, and their extensibility to higher dimensions, make them an attractive choice for Monte Carlo integration, e.g., in production rendering. Therefore, research interest has shifted to LD constructions with the main goal of improving their quality. For example, the famous rendering textbook, PBRT, gradually adopted LD and dropped blue-noise samplers [Pharr and Humphreys 2010; Pharr et al. 2016, Chapter 7].

While we believe that both research directions are important in their own right, here we aim to improve the quality of LD constructions. One way to improve LD sets and sequences is to combine the blue-noise property, defined in the frequency domain, and the low-discrepancy property, defined in the spatial domain. Although the possibility of combining the large Poisson-disk radius with the low discrepancy was pointed out early by Keller [2006], it was not until ten years later that Ahmed et al. [2016] presented a practical algorithm for imposing spectral control over LD sets. Their proof of concept showed that the two properties can coexist, inspiring other works that tried to combine these two sampling-pattern extremes, as we will review in the following section.

In this paper, we ask a more general question about what the design space for digital nets looks like. Is it feasible to incorporate additional quality criteria, such as a blue-noise frequency pattern? Specifically, we analyze $(0, m, 2)$-nets in base 2 , hereinafter called dyadic nets ${ }^{1}$ (DN), which deliver the lowest order of discrepancy. To this effect, we describe a novel construction algorithm that completely spans the design space and explicitly reveals the possible design choices. With this understanding, we can easily transform between DNs, optimize them to have particular properties, partition them, and upsample them. Our characterization also explains the limitations and degrees of freedom of the DNs. While the focus of our work is the analysis of nets and their construction, we also present some example applications, e.g., optimizing DNs to have blue-noise properties and generating multi-class DNs.

\subsection{Contributions}

In this paper, we present a comprehensive study of DNs that begins with their intuitive definition as multi-stratified sets and derives important properties, with the following outcomes:

(1) A hierarchical construction algorithm that spans all the possible DNs.

(2) A parameterization of the complete space of DNs. Each distinct $\mathrm{DN}$ can be mapped to a binary string, with each bit encoding a decision in the hierarchical construction algorithm, and bearing a geometric interpretation in the rendered net.

(3) An efficient algorithm for constructing a DN that tries to match a given stratified point set.

(4) An identification of the available degrees of freedom for transforming between nets that we exploit to design an efficient algorithm for generating optimized nets obeying a user-specified criterion.

(5) A derivation of an energy function for blue noise that we use to produce nets with high-quality blue noise.

(6) A theorem for upsampling nets that we use to show that the space of dyadic sequences is significantly smaller than that of nets, reducing the possibility of optimizing sequences.

\section{RELATED WORK}

In this section, we highlight the literature most relevant to our work, which may be grouped into three categories.

\subsection{Low-Discrepancy Nets and Sequences}

An LD point set is a point distribution that minimizes a measure called discrepancy [Zaremba 1968]. There are many variants of discrepancy. The most famous one is arguably the star discrepancy, $D^{*}$, which measures the maximum error in estimating the area of the rectangular region between the origin and an arbitrary point by calculating the ratio of points falling inside the rectangle. An LD sequence is a sequence of sample points that maintains a low discrepancy for all of its prefixes. Established discrepancy orders are $O\left(\log ^{s-1}(N) / N\right)$ and $O\left(\log ^{s}(N) / N\right)$ for $s$-dimensional point sets and sequences, respectively [Niederreiter 1992, Chapter 3].

Most LD sampling concepts and techniques were developed outside of computer graphics. Using bit reversal for generating welldistributed samples was introduced by van der Corput [1935] and

\footnotetext{
${ }^{1}$ Dyadic nets exist up to three dimensions [Dick and Pillichshammer 2010, Corollary
} 4.18 ], but in this paper, we only address $2 \mathrm{D}$ dyadic nets. 
initiated a whole line of research about LD sequences for quasiMonte Carlo sampling. Digital nets were introduced by Sobol [1967] for base- 2 and generalized by Niederreiter [1987], who introduced the $(t, m, s)$-net and $(t, s)$-sequence notations. Thorough discussions about nets and sequences may be found in Niederreiter [1992] and Dick and Pillichshammer [2010]. We need to clarify here that the term digital nets refers to the subset of nets that are generated using linear matrix operations applied to the sample index [Niederreiter 2005]. In this paper, we address a more general set of nets that we define in Section 3.4.

In computer graphics, LD sampling was introduced by Shirley [1991] to the best of our knowledge, followed by Mitchell [1992], and greatly advocated by Keller and colleagues [Grünschloß et al 2008; Grünschloß and Keller 2009; Grünschloß et al. 2012; Keller 2006, 2013; Kollig and Keller 2002]. An excellent introduction to LD sequences and their application to rendering is found in [Keller 2013].

\subsection{Blue Noise}

A blue-noise distribution is an isotropic, visually pleasing 2D distribution of points, characterized by a frequency power spectrum that has low energy in low frequencies, and a flat high-frequency "bluenoise" region [Ulichney 1988]. In contrast to LD sampling patterns, blue noise is an in-house product of the graphics community, which explains its popularity. The loose definition of blue noise in terms of its frequency power spectrum led to many algorithms for producing distributions that target that spectrum, including [Bridson 2007; Dunbar and Humphreys 2006; Ebeida et al. 2014, 2011, 2012; Eldar et al. 1997; Gamito and Maddock 2009; Jones 2006; Kensler 2013; McCool and Fiume 1992; Mitchell et al. 2018], and an equally long list of algorithms for optimizing a given set towards a blue-noise spectrum [Ahmed et al. 2017a; Balzer et al. 2009; Chen et al. 2012; Fattal 2011; de Goes et al. 2012; Heck et al. 2013; Jiang et al. 2015; McCool and Fiume 1992; Mitchell 1991; Öztireli and Gross 2012; Reinert et al. 2016; Schlömer et al. 2011; Zhou et al. 2012]. Many of these algorithms are energy-based, and therefore, may be adapted to work with our technique for optimizing dyadic nets. However, we note that our model is more suited to point-by-point energy functions, e.g., KDM [Fattal 2011], not global optimization, e.g., BNOT [de Goes et al. 2012].

As of this writing, we are unaware of an agreed-on mathematical theory on blue noise or a clear model for obtaining it. There are recent attempts, however, to fill this gap, e.g., [Heck et al. 2013; Öztireli 2020]. We will follow the works of [Fattal 2011; Hanson 2003, 2005; Óztireli et al. 2010; Ulichney 1993] and derive an energy expression for defining blue noise based on a Gaussian kernel.

\subsection{Low-Discrepancy Blue Noise}

A recent research trend was devoted to bridging the gap between the blue-noise and LD distributions [Keller et al. 2019]. Ahmed et al. [2016] presented the first algorithm for imposing spectral control over LD sets, using an original construction of the input sets. They showed that the LD quality of a set can be traded for a blue-noise spectrum at user-selected ratios. Perrier et al. [2018] extended the idea to sequences, though the generated sequences attained full optimization properties only at powers of 16 (typically) instead of 2 . Christensen et al. [2018] took a different path, using a back-tracking algorithm to search for good sequences, similar to an earlier work with nets by Grünschloß et al. [2008]. Pharr [2019] presented a more efficient search algorithm. These works are the most relevant to ours in the literature. We make the following step of showing that, at least for nets, a blue-noise spectrum can be incorporated without compromising the LD quality, thus realizing earlier speculation by Keller [2006]. We also lay a theoretical foundation for future research in this direction.

More recently, another trend appeared for producing a blue-noise distribution of the rendering error, rather than the input samples, using LD sampling patterns [Ahmed and Wonka 2020; Heitz and Belcour 2019; Heitz et al. 2019]. These techniques are orthogonal to our work, and we will point out how they may benefit from our method.

\section{ESSENTIAL BACKGROUND: DYADIC NETS AND SEQUENCES}

A stratified point set is obtained by partitioning the sampled domain regularly into non-overlapping small cells, called strata, and placing one or more sample points inside each stratum. In 2D, for example, a stratified point set of $N=n^{2}$ is obtained by partitioning the sampled domain into $n \times n$ strata. If $N$ is factorable in different ways, then we may also use different numbers of rows and columns. For example, if $N=b^{m}$ for some base $b$, then the domain may be partitioned into $b^{m} \times 1, b^{m-1} \times b, \cdots$, or $1 \times b^{m}$ strata. Such partitions are referred to as elementary intervals [Niederreiter 1992]. A net is a point distribution that maintains the stratification property over all such possible stratifications. Specifically, a so-called $(t, m, s)$-net in base $b$ is an $s$-dimensional point set of $b^{m}$ points that has exactly $b^{t}$ points inside each elementary interval of volume $b^{t-m}$, in every possible partitioning of the unit hypercube. Fig. 1 illustrates a few examples of $(0,6,2)$-nets in base 2 . A $(t, s)$-sequence in base $b$ is a sequence of sample points that is a $(t, m, s)$-net for all its $b^{m}$ prefixes.

The $t$ parameter indicates the quality of the distribution, with a smaller number corresponding to a higher quality. When $t=0$, we have only one sample point per stratum. Such $(0, m, s)$-nets are only possible for $s \leq b+1$ [Dick and Pillichshammer 2010, Corollary 4.18], and $(0, s)$-sequences are available only for $s \leq b$ [Dick and Pillichshammer 2010, Corollary 4.36]. In this paper, we are interested in the $2 \mathrm{D}$ binary case, that is, $(0, m, 2)$-nets and $(0,2)$-sequences in base 2 , which attain very low discrepancies.

\subsection{Generating Nets and Sequences}

Unlike its rival blue-noise distribution, the concept of nets and sequences have been primarily developed by mathematicians using algebraic equations rather than iterative processes. Specifically, a set of so-called generator matrices, one per axis, is employed to compute the components of a sample point from its sequence (index) number. In the binary case, the binary matrix is multiplied by a binary vector that holds the bits of the sample index in reverse order, i.e., least significant bit first. This digit reversal operation is fundamental in LD construction [Kuipers and Niederreiter 1974]. The resulting integer is divided by the number of points to obtain the 
coordinates. These matrices are carefully crafted and coordinated so as to enforce the desired partitioning properties. Different combinations of matrices can be used to obtain different distributions. Nets generated thus from matrices are called digital nets [Niederreiter 2005]. The simplest digital net is arguably the Hammersley point set, which employs two diagonal matrices:

$$
C_{x}=\left(\begin{array}{cccc}
0 & 0 & \cdots & 1 \\
0 & . & 1 & \vdots \\
\vdots & . & . & 0 \\
1 & \cdots & 0 & 0
\end{array}\right), \quad C_{y}=\left(\begin{array}{cccc}
1 & 0 & \cdots & 0 \\
0 & 1 & \ddots & \vdots \\
\vdots & \ddots & \ddots & 0 \\
0 & \cdots & 0 & 1
\end{array}\right) .
$$

Since the index bits are fed in reverse order, the inverse-diagonal matrix $C_{x}$ restores the points order, while the identity matrix $C_{y}$ generates a bit-reversed sequence.

The advantage of matrices is that they make it possible to deduce and tune the properties of the net by studying the generating matrices. For example, a $(0, m, 2)$-net property is guaranteed if all the hybrid matrices, obtained by appending the first $m-k$ rows of $C_{x}$ to the first $k$ rows of $C_{y}$, are full rank. This idea extends to constructing sequences. Specifically, Sobol's $(0,2)$-sequence is obtained using an infinite identity matrix for $\mathrm{x}$, and the infinite Pascal triangular matrix (2) for y:

$$
C_{1}=\left(\begin{array}{ccccccccc}
1 & 1 & 1 & 1 & 1 & 1 & 1 & 1 & \cdots \\
0 & 1 & 0 & 1 & 0 & 1 & 0 & 1 & \cdots \\
0 & 0 & 1 & 1 & 0 & 0 & 1 & 1 & \cdots \\
0 & 0 & 0 & 1 & 0 & 0 & 0 & 1 & \cdots \\
0 & 0 & 0 & 0 & 1 & 1 & 1 & 1 & \cdots \\
\vdots & \vdots & \vdots & \vdots & & \ddots & & &
\end{array}\right)
$$

\subsection{Scrambling Nets and Sequences}

Owen [1995] introduced a hierarchical axis-wise scrambling scheme that preserves the multi-stratification of nets. The idea is simple: The halves of the domain along each axis are arbitrarily swapped, and the swapping is applied recursively to these halves. The original goal of Owen's scrambling was to facilitate variance estimation in quasi-Monte Carlo integration, but it proved advantageous in improving both the convergence rate and the frequency spectrum, which is important in graphics applications.

A few variants of Owen's scrambling were proposed, primarily to reduce its computational complexity [Owen 2003]. The most notable is xor scrambling, which uses the same single swapping decision for the whole level in the hierarchical scrambling [Kollig and Keller 2002]. Applying this scrambling is as simple as xoring the computed coordinates against a single integer, which is of negligible cost. However, the resulting sets of xor scramblings of the same net are strongly correlated, leading to strong inter-pixel and interdimension correlations.

A closely related, but semantically different, scrambling is attributed to Faure and Tezuka [2002], who proposed applying an Owen-like scrambling to the sequence numbers of the samples. This basically alters the order of the samples in the produced sequence without changing the sample locations, which raises a question about the utility of such scrambling. Faure and Tezuka proposed it as an idea worth exploring, and an actual feasible application will be shown in Subsection 3.4 below.

\subsection{Optimizing Nets and Sequences}

The advantage of the aforementioned algebraic constructions is that they scale well with dimensions, when it becomes really difficult to visualize. One disadvantage is that the matrix-based constructions, even with Owen's scrambling, still do not encompass all possible nets, as we will see in the following section. There are more possibilities to explore, especially in the $2 \mathrm{D}$ case, that might have favorable properties, such as a blue-noise spectrum and/or a large Poisson-disk radius. As a proof of concept, Grünschloß et al. [2008] performed exhaustive search for matrix-based DNs with small point counts and exhibited nets with considerably large Poisson-disk radii. However, given the description above of dyadic nets, such a search is infeasible for large point sets, as the search space grows super-exponentially. In a follow-up paper, Grünschloß and Keller [2009] also presented non-matrix-based heuristic constructions that exhibit even larger Poisson-disk radii. Ahmed et al. [2016] presented an algorithm that starts with valid dyadic nets, but then compromises them to impose desirable spectral properties. Perrier et al. [2018] extended the idea to sequences, but with significantly degraded blue-noise quality. In Section 4, we reveal possible justifications for this difference between nets and sequences.

\subsection{Nets from Sequences}

A principle that goes back to Roth [Niederreiter 1988] may be used to construct $2 \mathrm{D}$ LD sets from $1 \mathrm{D}$ LD sequences, by pairing the coordinates from the sequence in one axis with a linearly ordered list $\{i / N\}_{i=0}^{N-1}$ of coordinates on the other axis. Ahmed et al. [2016] presented a more flexible construction that applies the sequences to point offsets along all rows and columns of a stratified set. In both constructions, the sequence(s) can be any LD sequence, but when the input is $(0,1)$-sequence(s) in base- 2 , the resulting set is a dyadic net. Our construction in the following section exploits this property.

One of the main advantages of Roth's or Ahmed's constructions is that, in addition to Owen's scrambling, they can employ FaureTezuka scrambling [2002], which reorders the input sequences, leading to a wider variety of nets. Our current research began when we rediscovered Faure-Tezuka scrambling while experimenting with Ahmed's construction. We also accidentally discovered an interesting net construction, obtained by using a Gray-code-ordered van der Corput (bit-reversed) sequence [Antonov and Saleev 1979] to provide point offsets along all rows and columns of a stratified set; see Fig. 2. This family of nets is characterized by large minimal spacing between the samples, and is superior in this aspect to the celebrated Larcher-Pillichshammer family of nets [Grünschloß et al. 2008; Grünschloß and Keller 2009; Larcher and Pillichshammer 2003]. The resulting nets are visually similar to those obtained by Grünschloß et al. [2008] via an exhaustive search over small nets $(m \leq 6)$, but are freely scalable and extremely fast to generate, since bit-reversal in Gray code is fast [Pharr et al. 2016]. Our generating code is provided in the supplementary materials. The Poisson-disk radius in these nets is provably $\sqrt[4]{3} / 2 \simeq 0.658$ of the hexagonal packing radius because 1) the same sequence of offsets is used along 


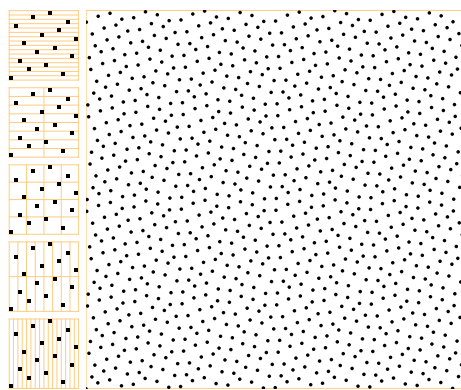

(a)

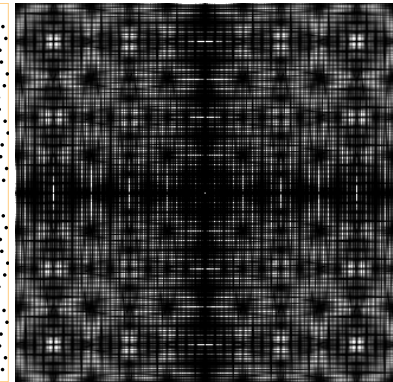

(c)

Fig. 2. A net construction obtained by using a bit-reversed sequence in a Gray-code ordering for the horizontal offsets along columns and the vertical offsets along rows of a stratified set. (a) A 16-point set, illustrating the $(0,4,2)$-net property. (b) A 1024-point set. (c) A periodogram of the 1024-point set.

all rows and columns (cf. CMJ [Kensler 2013]), hence points in the adjacent square strata are at least a stratum-width apart; and 2) the Gray-code ordering enforces diagonal neighbors to be at least a half-stratum apart along each axis. Discovering this net greatly encouraged our research, since it strongly suggested the existence of nets that approximate a blue-noise spectrum.

\section{OUR METHOD}

In this section, we present efficient algorithms for creating random and targeted nets, and for optimizing existing nets towards a prescribed energy-based configuration. Towards that end, we enumerate all possible nets up to a prescribed bit resolution $m$, and describe how to transform between them. This is made possible through a deep understanding of the structure of $2 \mathrm{D}$ nets and sequences that is difficult to derive starting from matrices. Therefore, we present an intuitive understanding of dyadic nets and sequences from first principles.

\subsection{Algebraic Formulation}

A $(0, m, 2)$-net in base 2 is basically a $2 \mathrm{D}$ set of $N=2^{m}$ points. The domain can be stratified into $N$ strata in $m+1$ ways:

$$
2^{m} \times 2^{0}, 2^{m-1} \times 2^{1}, \ldots, 2^{m-k} \times 2^{k}, \ldots, 2^{0} \times 2^{m},
$$

and these stratifications can be indexed by $k$, the bit resolution along the $y$-axis. Every stratum in each stratification must contain exactly one point to make a $(0, m, 2)$-net. This implies that each of the coordinates of points must have (at least) an $m$-bit resolution, encoded in binary as

$$
\begin{aligned}
& x_{i}={ }_{2} 0 . x_{i, 0} x_{i, 1} \cdots x_{i, m-1} \cdots, \\
& y_{i}=20 . y_{i, 0} y_{i, 1} \cdots y_{i, m-1} \cdots \text {. }
\end{aligned}
$$

We drop the point index suffix $i$ when implicitly understood and represent these coordinates as integers running from 0 to $N-1$, with an implicit denominator $N$. An example of a $(0,4,2)$-net containing 16 points is shown in Fig. 3.

The stratum index of a given point in the $k$ th stratification can be obtained from its $(x, y)$ coordinates by concatenating the most

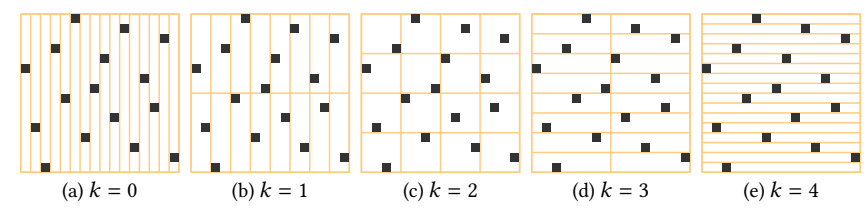

Fig. 3. A $(0,4,2)$-net in base 2 , having one point per stratum in all possible 16-strata regular partitionings of a square.

Table 1. Point coordinates and strata indices in binary for the set in Fig. 3, highlighting how they are related.

\begin{tabular}{|c|c|c|c|c|c|c|}
\hline \multicolumn{2}{|c|}{ Coordinates } & \multicolumn{5}{|c|}{ Stratum Index $q(x, y, k)$} \\
\hline $\mathrm{x}$ & $\mathrm{y}$ & $k=0$ & $k=1$ & $k=2$ & $k=3$ & $k=4$ \\
\hline 0000 & 1010 & 0000 & 1000 & 1000 & 1010 & 1010 \\
0001 & 0100 & 0001 & 0000 & 0100 & 0100 & 0100 \\
0010 & 0000 & 0010 & 0001 & 0000 & 0000 & 0000 \\
0011 & 1100 & 0011 & 1001 & 1100 & 1100 & 1100 \\
0100 & 0111 & 0100 & 0010 & 0101 & 0110 & 0111 \\
0101 & 1111 & 0101 & 1010 & 1101 & 1110 & 1111 \\
0110 & 0011 & 0110 & 0011 & 0001 & 0010 & 0011 \\
0111 & 1000 & 0111 & 1011 & 1001 & 1000 & 1000 \\
1000 & 1011 & 1000 & 1100 & 1010 & 1011 & 1011 \\
1001 & 0101 & 1001 & 0100 & 0110 & 0101 & 0101 \\
1010 & 1110 & 1010 & 1101 & 1110 & 1111 & 1110 \\
1011 & 0010 & 1011 & 0101 & 0010 & 0011 & 0010 \\
1100 & 1001 & 1100 & 1110 & 1011 & 1001 & 1001 \\
1101 & 0110 & 1101 & 0110 & 0111 & 0111 & 0110 \\
1110 & 1101 & 1110 & 1111 & 1111 & 1101 & 1101 \\
1111 & 0001 & 1111 & 0111 & 0011 & 0001 & 0001 \\
\hline
\end{tabular}

significant $m-k$ bits of $x$ to the most significant $k$ bits of $y$. Our convention is to use a row-major ordering. Algebraically, we define a function

$$
q(x, y, k)=y_{0} \cdots y_{k-1} x_{0} \cdots x_{m-k-1}
$$

to return the stratum index of a point $(x, y)$ in the $k$ th stratification. For example, Table 1 shows the strata indices for the set in Fig. 3. Given this function $q$, we have the following definition of dyadic 2D nets:

Definition 4.1. $A(0, m, 2)$-net is a point set such that, for each $k$ in $\{0, \ldots, m\}$, the set $\left\{q\left(x_{i}, y_{i}, k\right)\right\}_{i=0}^{N-1}$ of the point-associated strata is a full-rank permutation of the set $\{0, \ldots, N-1\}$.

\subsection{Generating Nets}

Rather than using matrices, we devise an efficient algorithm, Netshuffle, which directly populates the strata with points as outlined in Algorithm 1. Fig. 4 shows a visual illustration of the Netshuffle algorithm generating a $(0,4,2)$-net, Fig. 5 illustrates how it works, and Fig. 1(a) shows example nets produced by this algorithm.

The key to understanding the operation of the Netshuffle algorithm is that, as we move from the $k$ th to the $k+1$ st stratification, each pair of consecutive strata is merged vertically and split horizontally. To have one point in each stratum in the $k+1$ st stratification, we have to decide which of the two $x$ 's in the $k$ 's stratification is 


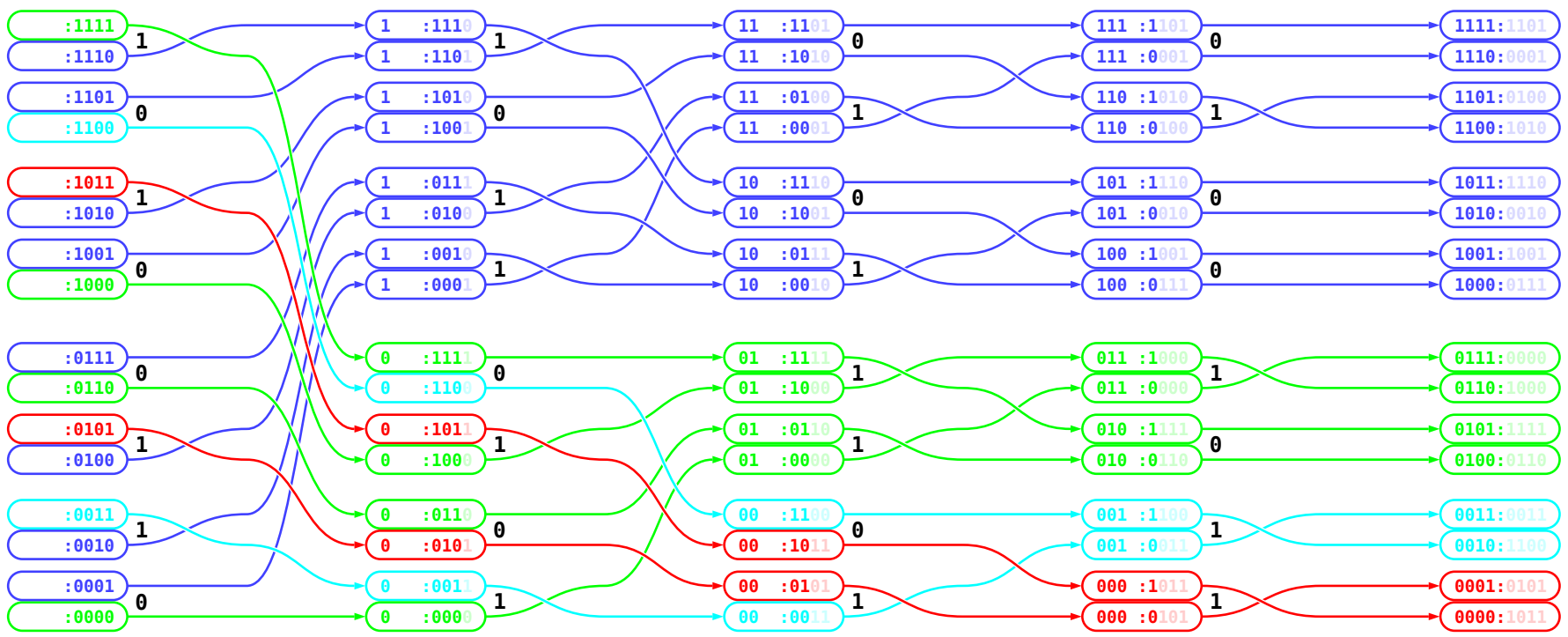

Fig. 4. A visual illustration of Algorithm 1 with $m=4$, which requires four steps of partitioning. The coloring is meant as a visual aid to see the flow. At the beginning, the $x$ 's are listed in their natural order, and as we move down the steps, the list is sorted by the paired $y$ 's, which are obtained by the step-wise reversal of the bits of $x$, optionally toggled for each pair of consecutive $x$ 's in the current list, as indicated by the large black digits. These toggle flags uniquely identify a net in the designated resolution. The position of the $x$-coordinate in each stage determines the index of the associated stratum in the respective stratification.

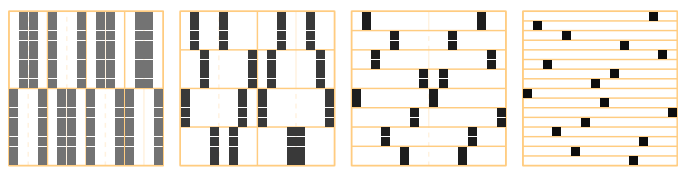

Fig. 5. A visual illustration of how Algorithm 1 gradually generates information about the paired $y$ 's of the given $x$ 's. It shows the same net as Fig. 4 with corresponding stages. At the beginning, we know nothing about the $y$ 's, and in each step, one more bit is decided on.

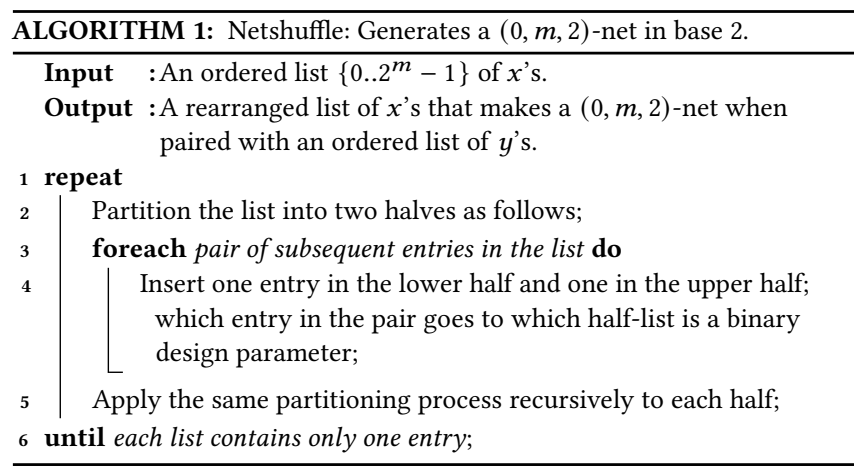

associated with the upper half and which with the lower half of the merged strata. This translates into a decision on the $k$ th bit of $y$ based on the $m-k-1$ st bit of the concerned $x$ 's. It does not matter which $x$ goes to which half; hence, the concerned bit may be xored with an optional binary bit, which we call a flag. The geometric implication of this flag is that a line connecting the two points may

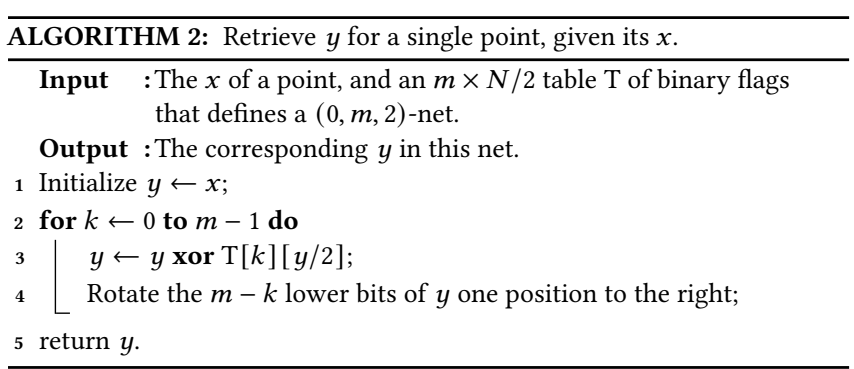

lean (0) forward or (1) backward because the smaller $y$ is paired with the (0) smaller or (1) larger $x$. This diagonal allocation of points is beneficial for integration, as noted by Öztireli [2016], because it poses negative correlations between the samples.

A second important insight into the Netshuffle algorithm is that the ordering of the strata changes as we move from the $k$ th to the $k+1$ st stratification. Therefore, the segregated $x$ 's have to be inserted $2^{m-k-1}$ slots apart.

The Netshuffle algorithm can be adapted to retrieve individual points, as outlined in Algorithm 2, for retrieving $y$ given $x$. A similar algorithm may be written for the reverse. However, the full table of flags must be known in order to retrieve an arbitrary point, since it is not known a priori how the coordinates are linked.

For a while, we mistakenly identified our new algorithm as a union between Owen's scrambling of the $x$ 's and Faure-Tezuka scrambling of the paired $y$ 's. After comparing the degrees of freedom, however, we realized that it is indeed a distinct novel algorithm. The Netshuffle algorithm offers $m \cdot N / 2$ bits that lead to distinct nets. We believe that these are all the available degrees of freedom 


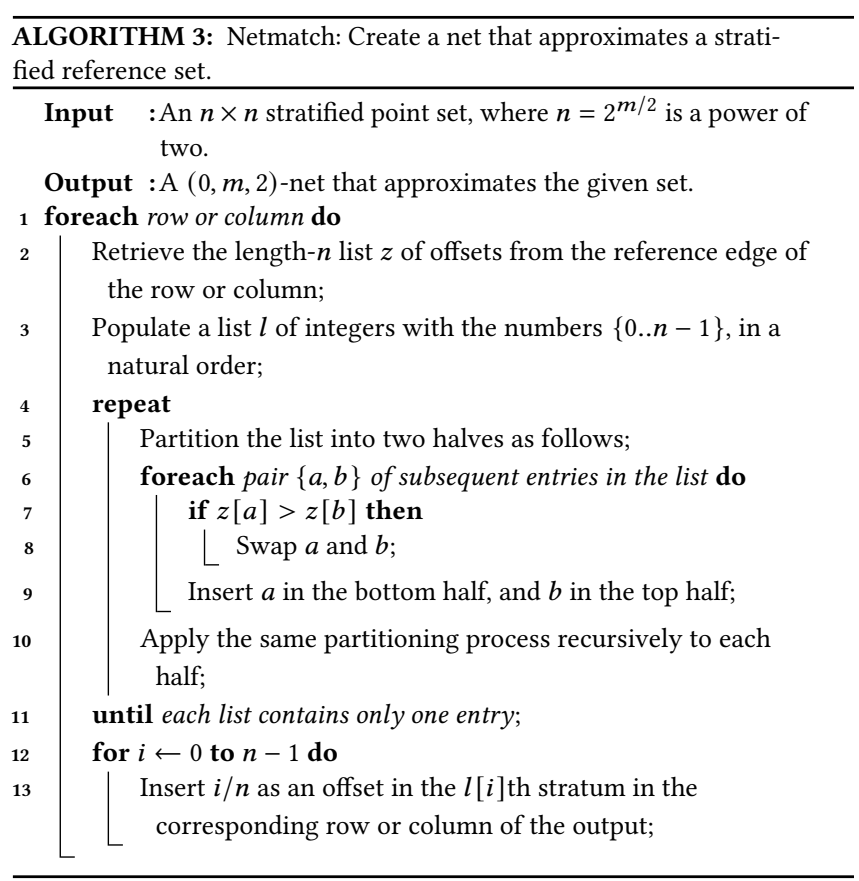

for constructing dyadic nets in 2D. Hence, the number of distinct $2 \mathrm{D}$ dyadic nets, using $m$ significant bits, is $2^{m \cdot N / 2}$. In contrast, the Owen's and Faure-Tezuka scramblings each offer $N-1$ bits for $N$-point nets, and these bits are inter-dependent. However, in all cases the sum is still below the capacity of Netshuffle. Thus, the number of settable bits is $O(N \log (N))$ for Netshuffle, compared to only $O(N)$ for combined Owen's and Faure-Tezuka scrambling.

Besides spanning the full set of nets, another advantage of the Netshuffle algorithm is that it enumerates (orders) the nets by concatenating all the bits for each binary design choice. An exhaustive search of nets, like in [Christensen et al. 2018; Grünschloß et al 2008; Pharr 2019], for example, is now straightforward, although intractable for $m=5$ (1.2 septillion nets) or larger. Of special interest are $(0,4,2)$-nets made up of 16 points, since they might be encoded in exactly one 32-bit unsigned integer, making it easy to manipulate and study these nets.

\subsection{Targeted Net Construction}

Instead of generating random nets, it may be desirable to generate nets that approximate a given reference distribution, as in LDBN [Ahmed et al. 2016]. Indeed, Algorithm 1 presents a way to generate $(0,1)$-sequences in one axis, and assign them to a linear list on the other axis, as per Roth's construction in Section 3.4. This immediately suggests adapting the algorithm for Ahmed's alternative construction, gaining more flexibility. Then, the algorithm can be further adapted to match a reference stratified set, as in LDBN, by producing a $(0,1)$-sequence that tries to match the sequence of offsets along rows and columns. Algorithm 3 outlines the steps. We see that the heart of the algorithm, lines $4-11$, is identical to the Netshuffle Algorithm 1, except that the swap decision is performed by comparing two values from the reference set.
In Fig. 6, two examples of matched references are presented and compared to LDBN [Ahmed et al. 2016]. As expected, LDBN is more faithful in matching the reference because it can permute over a 16-step resolution. On the other hand, our matched nets attain lower discrepancies. In the following subsection, we show that our model can attain better-quality targets than LDBN via direct optimization.

\subsection{Optimizing Nets}

Based on the insights from the construction algorithm, we can also derive the permissible operations that transform a valid DN into another valid DN. These transformations can then be used in an optimization framework that iteratively performs transformations in order to improve an objective function. First, we need the following definition:

Definition 4.2 (siblings). We call two strata in the same stratification siblings if their indices (Eq. (6)) share all but the least significant bit.

Then, we claim the following:

THEOREM 4.1. In any stratification of a dyadic net, the points in sibling strata can exchange their $y$-coordinates without destroying the net property.

That is, if the $x$-coordinate of each point in a pair of sibling strata is paired with the $y$-coordinate of the other point, the net property is preserved. While this key property of digital nets is very simple, it significantly contributes to the understanding of digital nets and their construction.

Proof. Let $\left(x_{i}, y_{i}\right)$ and $\left(x_{j}, y_{j}\right)$ be two points that reside in a pair of sibling strata in the $k$ th stratification, and let $k<m$. The strata indices can be obtained using Eq. (6):

$$
\begin{aligned}
q\left(x_{i}, y_{i}, k\right) & =y_{i, 0} \cdots y_{i, k-1} x_{i, 0} \cdots x_{i, m-k-1}, \\
q\left(x_{j}, y_{j}, k\right) & =y_{j, 0} \cdots y_{j, k-1} x_{j, 0} \cdots x_{j, m-k-1} .
\end{aligned}
$$

Given that the strata are siblings and $k<m$, Eqs. $(7,8)$ imply

$$
\begin{aligned}
y_{i, 0} \cdots y_{i, k-1} & =y_{j, 0} \cdots y_{j, k-1}, \\
x_{i, 0} \cdots x_{i, m-k-2} & =x_{j, 0} \cdots x_{j, m-k-2} .
\end{aligned}
$$

After swapping the $y$ 's, the new points become $\left(x_{i}, y_{j}\right)$ and $\left(x_{j}, y_{i}\right)$. Their associated strata in an arbitrary stratification $k^{\prime}$ read:

$$
\begin{aligned}
& q\left(x_{i}, y_{j}, k^{\prime}\right)=y_{j, 0} \cdots y_{j, k^{\prime}-1} x_{i, 0} \cdots x_{i, m-k^{\prime}-1}, \\
& q\left(x_{j}, y_{i}, k^{\prime}\right)=y_{i, 0} \cdots y_{i, k^{\prime}-1} x_{j, 0} \cdots x_{j, m-k^{\prime}-1} .
\end{aligned}
$$

If $k^{\prime} \leq k$, then Eq. (9) implies that all the leading $y$-bits in Eqs. (11, 12) are identical; hence,

$$
\begin{aligned}
& q\left(x_{i}, y_{j}, k^{\prime}\right)=q\left(x_{i}, y_{i}, k^{\prime}\right), \\
& q\left(x_{j}, y_{i}, k^{\prime}\right)=q\left(x_{j}, y_{j}, k^{\prime}\right) .
\end{aligned}
$$

When $k^{\prime}>k$, Eq. (10) implies that all the trailing $x$-bits in Eqs. (11, 12) are identical; hence,

$$
\begin{aligned}
& q\left(x_{i}, y_{j}, k^{\prime}\right)=q\left(x_{j}, y_{j}, k^{\prime}\right), \\
& q\left(x_{j}, y_{i}, k^{\prime}\right)=q\left(x_{i}, y_{i}, k^{\prime}\right) .
\end{aligned}
$$




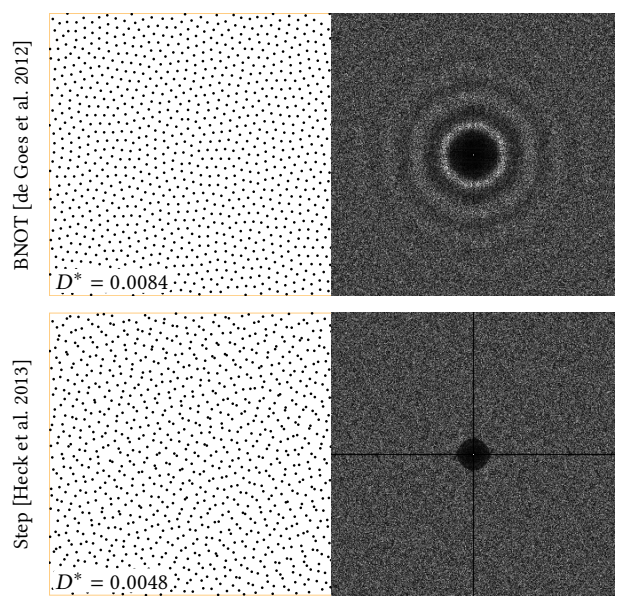

(a) Stratified Reference

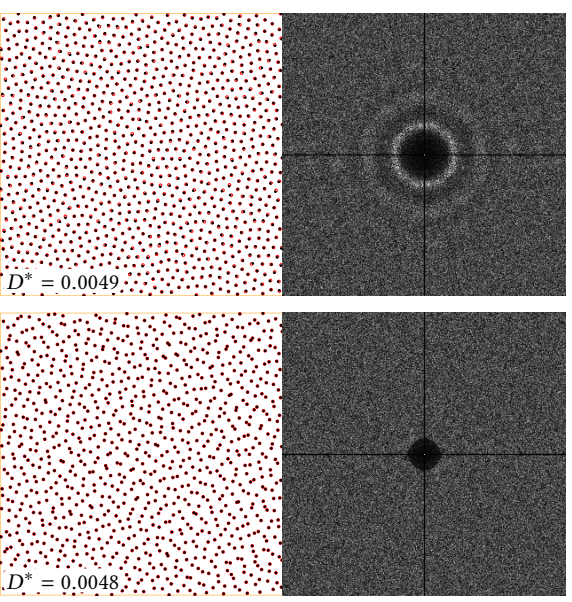

(b) LDBN [Ahmed et al. 2016]

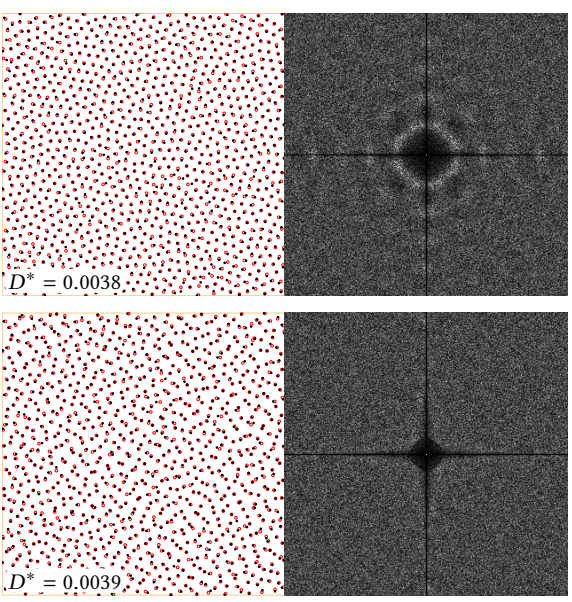

(c) Netmatch (Ours)

Fig. 6. Output of our Netmatch Algorithm 3 using (top) a stratified BNOT and (bottom) a Latinized and stratified step set. LDBN [Ahmed et al. 2016] is shown for comparison. The reference points are shown in red in (b) and (c). Note that Netmatched sets are less faithful in matching the target, but exhibit the characteristic spectrum of dyadic nets along the axes.

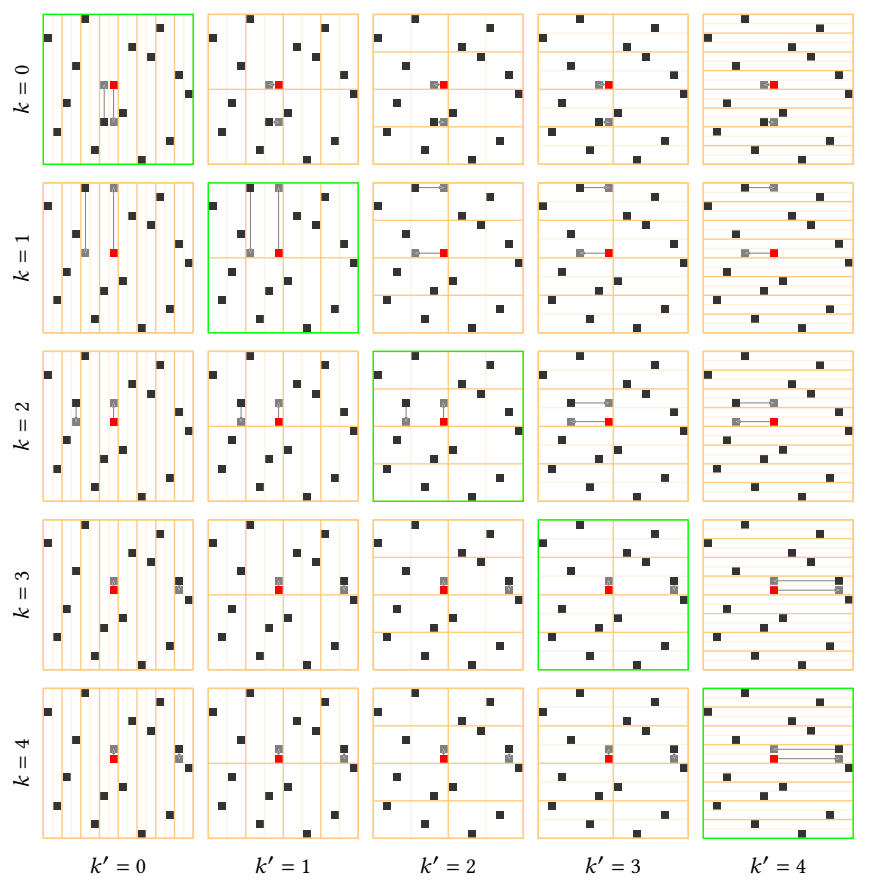

Fig. 7. Swap candidates of a point in a $(0,4,2)$-net, based on (rows) different stratifications, and their effect shown for (columns) all the stratifications The reference point is shown in red, and the new positions of the swapped points are shown in gray. The green outline indicates the stratification in which the swapping decision is made. Note that for all swaps, the moved points remain inside their strata in all stratifications. Note also that the swaps in the fourth stratification are identical to the third because every pair of sibling strata contains the same points as a corresponding pair there.

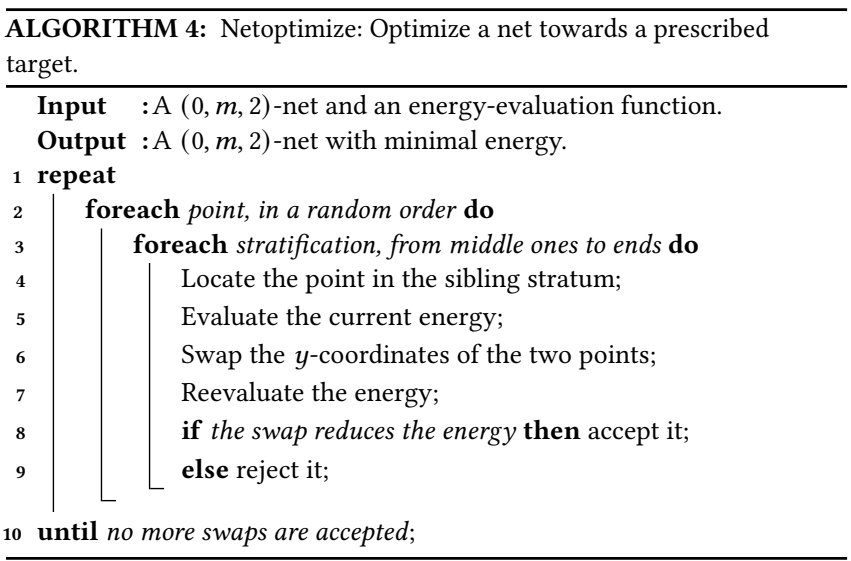

Thus, following the swap, the two points remain inside their strata in all stratifications, only slid vertically for $k^{\prime} \leq k$ and horizontally for $k^{\prime}>k$, as illustrated in Fig. 7 .

Finally, when $k=m$, the strata are indexed by $y$. If the strata are siblings, then $y_{i}$ differs from $y_{j}$ only in the least significant bit, which makes the points associated with sibling strata in the $m-1$ st stratification as well; otherwise, we would have the two points sharing the same stratum. Therefore, all the possible swaps in the $m$ th stratification are covered in the $m-1$ st stratification.

Converting the preceding observation to an optimization algorithm is straightforward, as outlined in Algorithm 4 and illustrated in Fig. 8. We iterate through the points and stratifications, starting from the stratifications with more compact strata $(k \simeq m / 2)$ and moving towards ones with narrower strata. For each stratification, we identify the swap-candidate point, which resides in a sibling stratum, and apply a swap if it is beneficial towards an optimization criterion. The process is repeated until no more swaps can be carried 


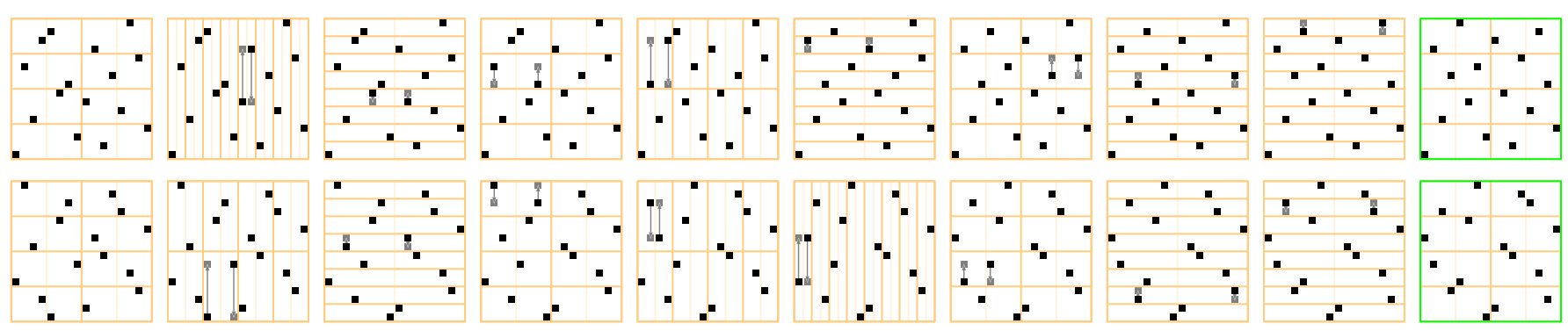

Fig. 8. A demonstration of our net optimization Algorithm 4, starting from random $(0,4,2)$-nets and optimizing towards (top) wider spacing and (bottom) denser clustering of the points. The leftmost plot is the initial net, the middle plots show the swapping steps, and the final net is shown with a green outline in the rightmost plot

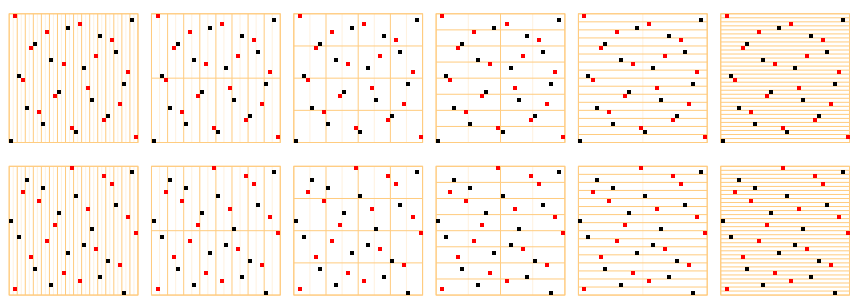

Fig. 9. Examples of complement nets of (top) a Hammersley net, and (bottom) a random net. Points of the reference 16-point net are shown in black, and the complement net is shown in red.

out. There is more than one way to locate the candidates. The most straightforward way is to maintain lists of pointers from all strata to points, and update them whenever a swap takes place. According to actual execution times, however, we found it more efficient to scan the whole row or column and identify the matching points.

Possibly, the most obvious optimization criterion is maximizing the Poisson-disk radius. Fig. 1(b) shows examples of nets optimized towards this target. Starting from a random net, the resulting point sets and their frequency power spectra are pretty similar to the Poisson-disk distributions obtained by dart throwing [Cook 1986; Dippé and Wold 1985]. Their average Poisson-disk radius (0.74) is in the typical range [Lagae and Dutré 2008], but our sets enjoy the very low discrepancy of $(0, \mathrm{~m}, 2)$-nets. In Section 5 , we present a more sophisticated energy function for inducing better blue-noise frequency spectra.

\subsection{Upsampling and Factoring}

Having a net that can be factored into smaller nets or incremented to a larger net is quite desirable and a main motivation behind recent literature on this topic [Christensen et al. 2018; Pharr 2019]. One of the most important results of our research is the identification of the possibilities and limitations of this idea. Specifically, we have the following theorem:

Theorem 4.2. For any given $(0, m, 2)$-net $A$ in base 2 , there exists one and only one $(0, m, 2)$-net, $\bar{A}$, that complements it into a $(0, m+1,2)$-net, obtained by complementing all the flags.

Fig. 9 shows examples of complement nets. A sketch of a proof of Theorem 4.2 starts by noting that, for any pair of sibling strata in a stratification $k<m$, the associated points reside in diagonally opposite quadrants of the shared space because they must be on different sides of both the vertical splitting line of the $k$ th stratification and the horizontal splitting line of the $k+1$ st stratification. The corresponding points in a net with complement flags, by definition, have to occupy the quadrants on the opposite diagonal. We note that such quadrants in the $k$ th stratification of a $(0, m, 2)$-net make the strata in the $k+1$ st stratification of a $(0, m+1,2)$-net. This proves that all strata contain one and only one point in the $(0, m+1,2)$-net. Finally, the first and last stratifications are handled separately by sharing the complementing least-significant bits between corresponding points of the complementing nets.

Theorem 4.2 is somewhat disappointing with respect to recent research efforts looking for good sequences [Christensen et al. 2018; Pharr 2019]. It tells us that, once $N=2^{m}$ points are fully decided, the locations of the subsequent $N$ points are also fully determined up to the $m$ th bit. Since we distinguish a $(0, m, 2)$-net by the most significant $m$ bits of the point coordinates, the degrees of freedom in upsampling a given distinct net are only in the least significant bits beyond the $m-1$ st. Thus, out of the $(m+1) \times N$ binary dimensions of distinct $(0, m+1,2)$-nets, only $N$ correspond to nets that can be factored into two dyadic nets. The majority of dyadic nets are prime, meaning that they cannot be factored into two dyadic nets. In other words, the space of $(0,2)$-sequences is significantly lower dimensional than that of nets. Nevertheless, there is still an exponentially growing number of distinct sequences. We also note that useful sequences do not necessarily have to adhere to the definition and start with one point.

When it comes to optimization, to preserve the factors we can only swap the points in the first and last stratifications of a composite net. This coincides with the leaf level of an Owen's scrambling tree. By induction from a $(0,0,2)$-net (one point), we conclude that Owen-scrambled Sobol sequences are the one and only set of dyadic $(0,2)$-sequences, though we do not have a formal proof of this insight.

We were hoping that optimizing a net would automatically optimize its complement, but that is not the case, as illustrated in Fig. 10(a). It is possible, however, to jointly optimize the two nets together by adapting Algorithm 4 to evaluate the energy gain and loss in both nets. Fig. 10(b) shows an example of joint optimization. It is 


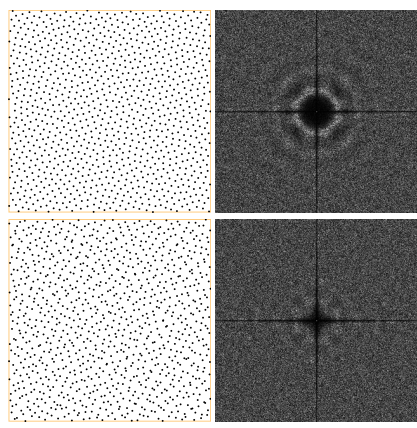

(a) Only top net optimized

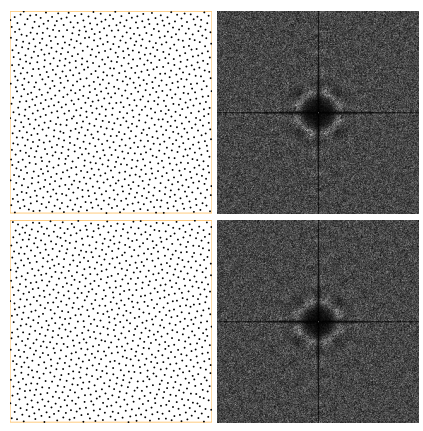

(b) Both optimized jointly
Fig. 10. Optimizing complement nets for blue noise. (a) The net in the top row is optimized independently to acquire high-quality blue noise. The complement net looks random. (b) Both nets are optimized jointly, acquiring modest blue-noise quality.

also possible to optimize the union net as well, thus creating a multiclass net [Wei 2010]. In theory, it is also possible to optimize across two or more generations to create an optimized sequence. Apart from the growing coding complexity, however, we also point out that joint optimization poses more constraints that reduce the chance of finding beneficial swaps, which is another way to see the difficulty of having an optimized sequence. Indeed, the unconstrained optimization of the single net in Fig. 10(a) leads to significantly better quality than the jointly optimized complementing nets in Fig. 10(b).

\section{BLUE-NOISE OPTIMIZATION}

Most of the algorithms that produce blue-noise distributions were presented in their original papers as approximating or close to the blue-noise distribution, but no specific method claims to be the reference blue noise that is being approximated. More specifically, we could not identify a specific criterion to use as an energy function for evaluating the blue-noise quality of a distribution. Indeed, the Poisson-disk radius, centroidal Voronoi energy, and equal Voronoior power-cell capacities, among others, were all proposed as measures, but we are unaware of an agreed-on measure. In this section, we derive from first principles what we think is a good mathematical definition of blue noise, and highlight its relation to common measures and distributions.

We start from the basic description of blue noise as an even, featureless, eye-pleasing distribution of points [Ulichney 1987, 1988]. Looking from a distance at a blue-noise distribution of black dots, one should see nothing but a constant level of grey because displaying the sample points is equivalent to sampling a DC function. Approximating the eye function by Gaussian filtering, as noted in [Stoppel and Bruckner 2019], a blue-noise distribution may be modeled as a distribution that minimizes the variance of a field of Gaussian kernels placed at the points. A similar model was first presented by Hanson [2003; 2005] - to the best of our knowledge and generalized by Fattal [2011]. Both authors, however, continued with numerical solutions. Here, we proceed to give an analytical solution.

\subsection{Derivation}

Let

$$
\mathbf{P}=\left\{\mathbf{x}_{i}\right\}_{i=1}^{N}
$$

be a set of $N$ 2D points,

$$
\mathbf{x}_{i}=\left(x_{i}, y_{i}\right),
$$

approximating a uniform density in a toroidal domain $D$. For simplicity, we assume that each point accounts for a unit area. Thus, the domain area is $N$, and its length is $\sqrt{N}$ along each dimension. By placing a Gaussian at each point, we have the density function

$$
A(\mathbf{x})=\sum_{i=1}^{N} \exp \left(-\frac{\left\|\mathbf{x}-\mathbf{x}_{i}\right\|^{2}}{2 \sigma^{2}}\right),
$$

which should closely approximate the average gray level everywhere. Therefore, we want to minimize the variance

$$
\operatorname{Var}(A(\mathbf{x}))=E\left((A(\mathbf{x}))^{2}\right)-(E(A(\mathbf{x})))^{2} .
$$

Up to this point, the model is identical to [Fattal 2011], but we proceed differently. The second term is fixed, so our goal is to minimize the average square

$$
E\left((A(\mathbf{x}))^{2}\right)=\frac{1}{N} \int_{D} A^{2}(\mathbf{x}) d \mathbf{x}
$$

of $A(\mathbf{x})$. We proceed by expanding the integrand

$$
\begin{aligned}
A^{2}(\mathbf{x}) & =\left(\sum_{i=1}^{N} \exp \left(-\frac{\left\|\mathbf{x}-\mathbf{x}_{i}\right\|^{2}}{2 \sigma^{2}}\right)\right)^{2} \\
& =\sum_{i=1}^{N} \sum_{j=1}^{N} \exp \left(-\frac{\left\|\mathbf{x}-\mathbf{x}_{i}\right\|^{2}}{2 \sigma^{2}}\right) \exp \left(-\frac{\left\|\mathbf{x}-\mathbf{x}_{j}\right\|^{2}}{2 \sigma^{2}}\right) \\
& =\sum_{i=1}^{N} \sum_{j=1}^{N} \underbrace{\exp \left(-\frac{\left\|\mathbf{x}-\mathbf{x}_{i}\right\|^{2}+\left\|\mathbf{x}-\mathbf{x}_{j}\right\|^{2}}{2 \sigma^{2}}\right)}_{g_{i, j}(\mathbf{x})} .
\end{aligned}
$$

With some algebraic manipulation, shown in Appendix A, we arrive at

$$
A^{2}(\mathbf{x})=\sum_{i=1}^{N} \sum_{j=1}^{N} \exp \left(-\frac{\left\|\mathbf{x}_{i}-\mathbf{x}_{j}\right\|^{2}}{4 \sigma^{2}}\right) \exp \left(-\frac{\left\|\mathbf{x}-\frac{\mathbf{x}_{i}+\mathbf{x}_{j}}{2}\right\|^{2}}{\sigma^{2}}\right) .
$$

Substituting this in Eq. (21) gives

$$
\begin{array}{r}
E\left((A(\mathbf{x}))^{2}\right)=\frac{1}{N} \sum_{i=1}^{N} \sum_{j=1}^{N} \exp \left(-\frac{\left\|\mathbf{x}_{i}-\mathbf{x}_{j}\right\|^{2}}{4 \sigma^{2}}\right) \\
\cdot \int_{D} \exp \left(-\frac{\left\|\mathbf{x}-\frac{\mathbf{x}_{i}+\mathbf{x}_{j}}{2}\right\|^{2}}{\sigma^{2}}\right) d \mathbf{x} .
\end{array}
$$

Assuming a sufficiently large number of samples to make the kernel support much smaller than the domain length, i.e.,

$$
\sigma \ll \sqrt{N}
$$


or, alternatively, assuming an infinite periodic set, then the Gaussian integral evaluates to

$$
\begin{aligned}
\int_{D} \exp \left(-\frac{\left\|\mathbf{x}-\frac{\mathbf{x}_{i}+\mathbf{x}_{j}}{2}\right\|^{2}}{\sigma^{2}}\right) d \mathbf{x} & =\int_{-\infty}^{\infty} \int_{-\infty}^{\infty} \exp \left(-\frac{\left\|\mathbf{x}-\frac{\mathbf{x}_{i}+\mathbf{x}_{j}}{2}\right\|^{2}}{2(\sigma / \sqrt{2})^{2}}\right) d \mathbf{x} \\
& =2 \pi \frac{\sigma^{2}}{2} \\
& =\pi \sigma^{2}
\end{aligned}
$$

Therefore,

$$
E\left((A(\mathbf{x}))^{2}\right)=\frac{\pi \sigma^{2}}{N} \sum_{i=1}^{N} \sum_{j=1}^{N} \exp \left(-\frac{\left\|\mathbf{x}_{i}-\mathbf{x}_{j}\right\|^{2}}{4 \sigma^{2}}\right) .
$$

Similarly,

$$
E(A(\mathbf{x}))=\frac{1}{N} \sum_{i=1}^{N} \int_{D} \exp \left(-\frac{\left\|\mathbf{x}-\mathbf{x}_{i}\right\|^{2}}{2 \sigma^{2}}\right) d \mathbf{x}=2 \pi \sigma^{2} .
$$

Substituting Eqs. $(31,32)$ in Eq. (20) gives

$$
\operatorname{Var}(A(\mathbf{x}))=\frac{\pi \sigma^{2}}{N} \sum_{i=1}^{N} \sum_{j=1}^{N} \exp \left(-\frac{\left\|\mathbf{x}_{i}-\mathbf{x}_{j}\right\|^{2}}{4 \sigma^{2}}\right)-\left(2 \pi \sigma^{2}\right)^{2} .
$$

The normalized standard deviation of the filtered set is

$$
\mathrm{SD}(A(\mathbf{x}))=\sqrt{\frac{1}{N} \sum_{i=1}^{N} \sum_{j=1}^{N} \frac{1}{\pi \cdot 4 \sigma^{2}} \exp \left(-\frac{\left\|\mathbf{x}_{i}-\mathbf{x}_{j}\right\|^{2}}{4 \sigma^{2}}\right)-1 .}
$$

We note that the summand is a normalized Gaussian whose variance $\left(\sigma^{2}\right)$ is twice the one used for filtering.

A geometric interpretation of Eq. (34) is that the normalized variance is the average sampling error of a normalized Gaussian with doubled variance, centered at each sample point, and sampled by the others. We note that by the definition of variance, this average has to be positive, irrespective of how the samples are distributed. It is maximized when all the samples are at one point. There are important insights we gain from this formulation. First, since the Gaussian function is radially symmetric, the variance of the filtered samples depends only on the absolute distances between pairs of samples. Secondly, due to the exponential decay of the Gaussian function, only points close to each point contribute significantly to the variance, and it is desirable to push the points away from the peak of the curve. This gives a justification for the classical wisdom of Poisson-disk sampling. By combining these two observations, we see that the dependence of variance on the area coverage is only indirect, via the need for maximizing the spacing of the samples. This formulation also explains the wide range of distributions that approximate blue noise. For example, instead of maximizing the mutual distance among all the points, it is also possible to push the points further apart, at the cost of having one close neighbor of each point.

Placing Gaussians at the points may be seen as a convolution with a Gaussian filter, which transforms into a multiplication by a corresponding Gaussian in the frequency domain. Reducing the variance in the spatial domain is equivalent to leaving only a DC peak and a low level of noise in the frequency domain. Since the energy of the Gaussian filter is concentrated in the low-frequency range, the frequency spectrum of a variance-reducing distribution has to be low in this range, which explains the characteristic frequency power spectrum of blue noise.

\subsection{Optimization}

Translating Eq. (34) into an energy-based optimization algorithm is straightforward. First, we isolate the contribution of an individual point to the variance term in Eq. (33):

$$
\operatorname{Var}\left(\mathbf{x}_{i}\right) \propto \sum_{j=1}^{N} \exp \left(-\frac{\left\|\mathbf{x}_{i}-\mathbf{x}_{j}\right\|^{2}}{4 \sigma^{2}}\right) .
$$

Then, we simply need to move the points in the direction that reduces this sum. Applying this idea to net optimization is straightforward. We iterate through the sibling strata, evaluating the sum of the energy of the associated points before and after a swap, and accepting the swap if the sum is reduced. Such an algorithm is strictly descending. The global minimum is reached when each point resides in the trough of the field of Gaussians placed at the other points, which is exactly the target of Ulichney's void-and-cluster algorithm [1993]. Remarkably, Öztireli et al. [2010] also suggested a gradient-descent algorithm with a Gaussian kernel similar to this, but they derived it differently. Thus, Eq. (34) underlies many of the blue-noise algorithms, including dart throwing [Cook 1986; Dippé and Wold 1985], Hanson [2003; 2005], and Fattal's Kernel Density Model (KDM) [2011].

As we noted earlier, the standard deviation of the Gaussian used for optimization is different from that used for filtering. Since we are interested in the former, we redefine

$$
\sigma \leftarrow 2 \sigma_{\text {Filtering }},
$$

so that the summands in the energy terms are similar to normalized Gaussians. It is worthwhile having a look at the influence of this $\sigma$ parameter. We note that the narrower the filter in the spatial domain, the wider its image in the frequency domain, and vice versa. Hence, narrow kernels produce a wider-band blue noise with more residual noise in low frequency, while wider filters enforce lower energy in the low frequencies, that is, global uniformity, at the cost of having a smaller low-energy band, as can be seen in Fig. 1(c, d, e).

\section{RESULTS}

In this section, we discuss the practical aspects of our modeling and algorithms, and compare them to the most relevant alternatives in each aspect. In the following discussion, Blue Nets refer to bluenoise-optimized nets, as described in Section 5.2, using $\sigma=0.5$.

\subsection{Blue-Noise Properties}

Fig. 11 compares Blue Nets to state-of-the-art randomized/optimized LD constructions, including Owen-scrambled Sobol sequence [Owen 1995, 1998], low-discrepancy blue-noise (LDBN) sets [Ahmed et al. 2016], blue-noise low-discrepancy sequence (BNLD), [Perrier et al. 2018], and progressive multi-jittered $(0,2)$ blue-noise sequences (PMJ02BN). Among these four, Owen-scrambled Sobol and PMJ02BN are proper dyadic nets. LDBN is not a dyadic net. It starts with a 

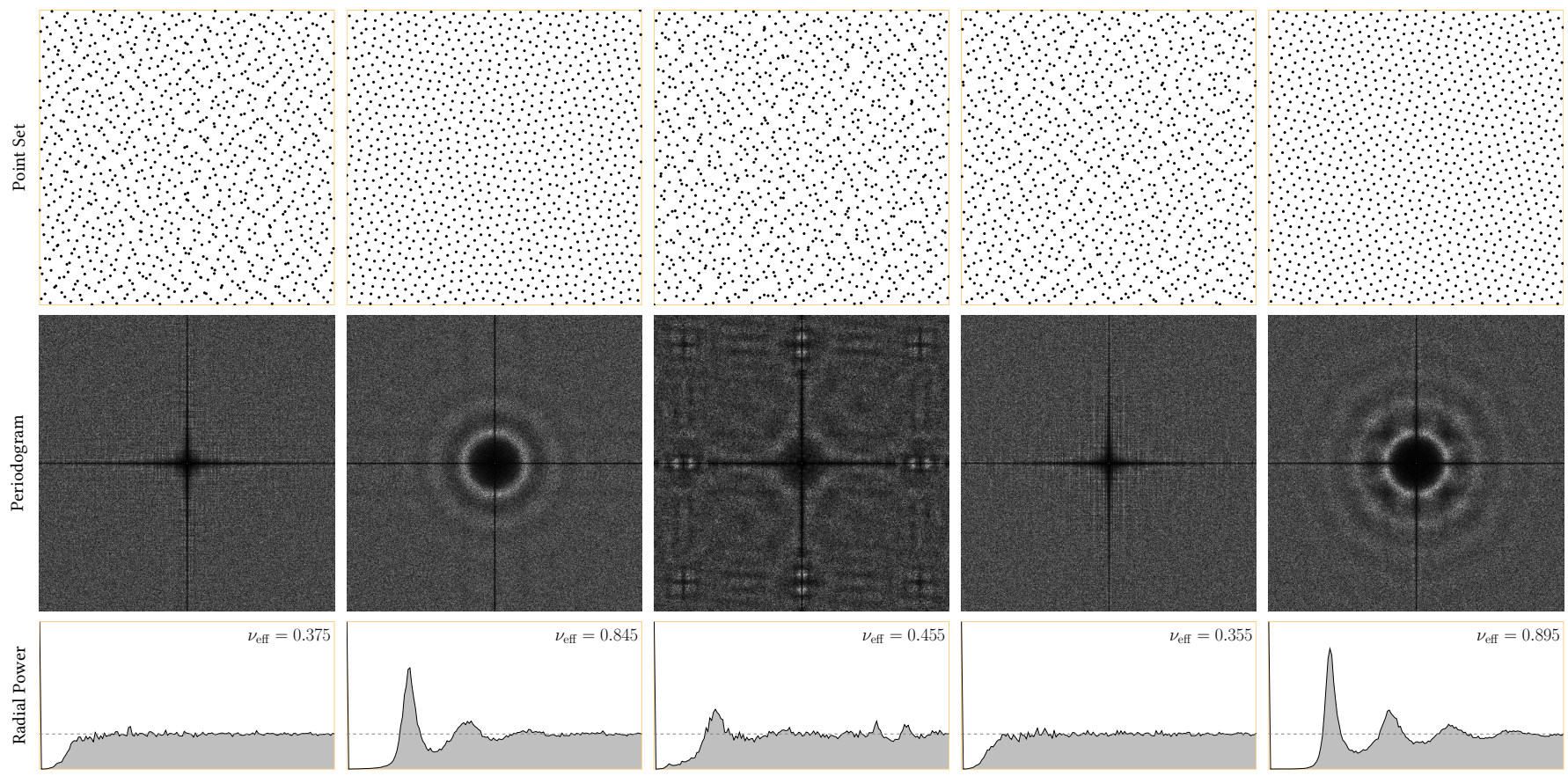

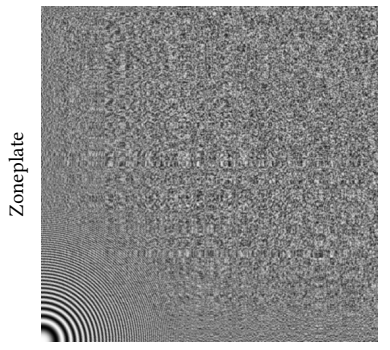

(a) Owen Scrambling[Owen 1995

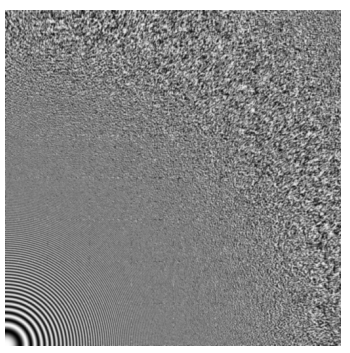

(b) LDBN [Ahmed et al. 2016]

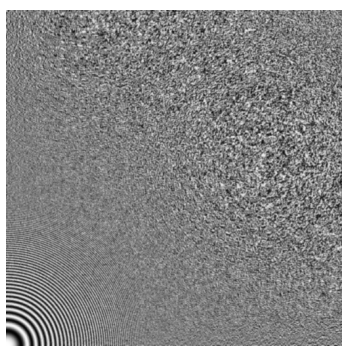

(c) BNLD [Perrier et al. 2018]

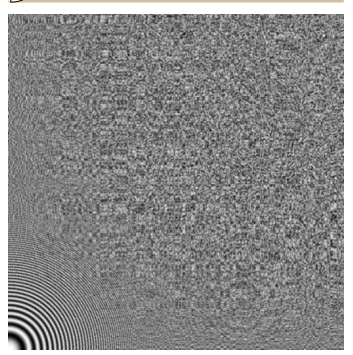

(d) PMJ02BN [Christensen et al. 2018]

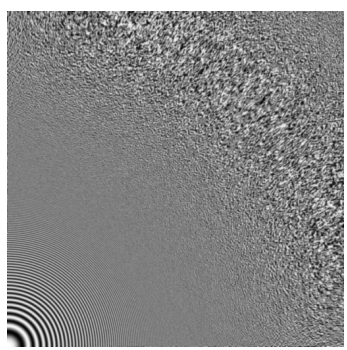

(e) Blue Nets (Ours)

Fig. 11. Comparing our Blue Nets to state-of-the-art randomized/optimized low-discrepancy distributions, showing different aspects. The point set in the top shows 1024 points, the other plots use 4096 points. The $v_{\text {eff }}$ value in the radial power plots is the effective Nyquist rate, as defined in [Heck et al. 2013], which measures the effective bandwidth of recoverable frequency contents. This is visually reflected in the zoneplate plots.

dyadic net, namely the Hammersley set, and compromises the quality factor $t$ of the net to incorporate blue-noise properties. BNLD, according to authors [Coeurjolly and Perrier 2019], attains its blue noise and dyadic properties only with $K^{2 n}$ samples. So for $K=4$, 1024 is not a multiple of $4^{2 n}$, whereas $4096=4^{6}$ is.

Thanks to the direct optimization capability, our Blue Nets are superior in blue-noise quality, surpassing LDBN, while still retaining their dyadic stratification. Indeed, our point sets exhibit the largest Poisson-disk radius, and the widest low-energy zone, which is reflected in the low-noise range of the zoneplate plot.

\subsection{Discrepancy}

Since our Netshuffle algorithm spans all possible dyadic nets, there is not much point in comparing the discrepancy of nets generated by this algorithm to other generation methods. It is interesting, however, to see the effect of blue-noise optimization on discrepancy, if any. In Fig. 12, we compare the star discrepancy [Zaremba 1968] of Blue Nets to some well-known nets. For each size of net in this test, we generated many realizations and selected the one with the largest Poisson-disk radius.

Hammersley sets are known to have the worst star discrepancy among nets [Larcher and Pillichshammer 2003], while LarcherPillichshammer nets are the best - to our knowledge. We note in Fig. 12 that Blue Nets seem to be in the middle, possibly closer to the lower bound, and notably superior to an unscrambled Sobol sequence. While this is not yet sufficient evidence to conclude a negative correlation between blue noise and discrepancy, it is apparent that the two properties are not positively correlated. That is, if blue-noise optimization does not improve the uniformity, as measured by discrepancy, it does not worsen it.

\subsection{Time and Space Complexity}

The time complexity of the Netshuffle Algorithm 1 is $O(N \log (N))$, which is optimal in the sense that this is the actual dimensionality 


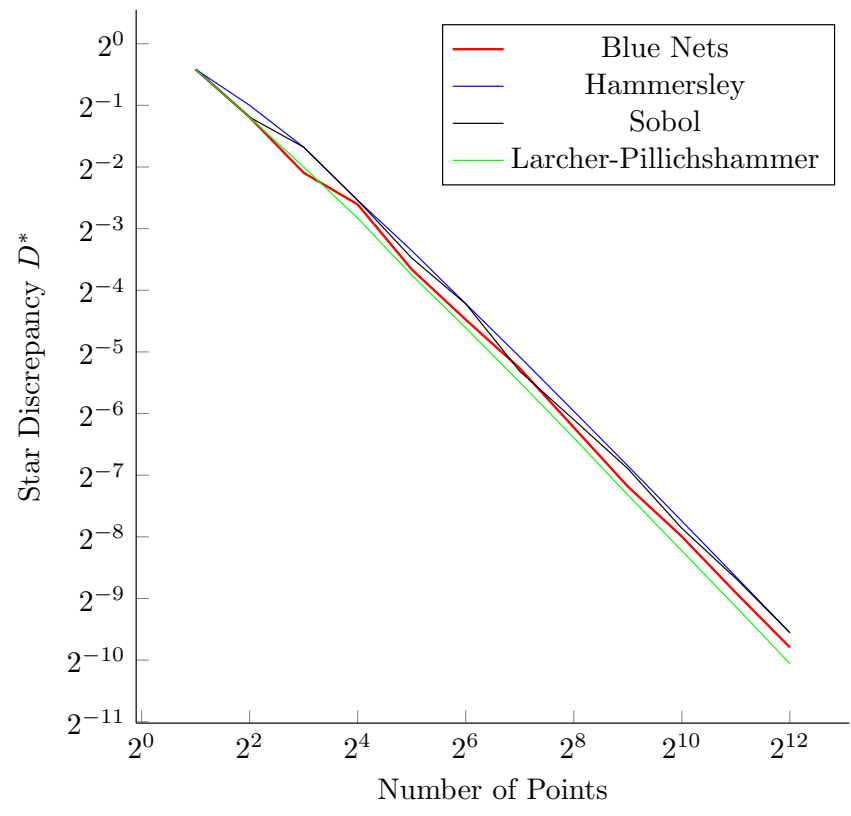

Fig. 12. Discrepancy comparison of blue-noise-optimized nets to wellknown dyadic distributions.

of the net space. The time complexity of the point-by-point Algorithm 2 is $O(\log (N))$, the same order as, say, computing a Sobol or a van der Corput sequence, though the partial bit rotation adds a few more operations. This means that, if the flags are already cached, random nets can be generated very quickly with our Netshuffle algorithm; possibly faster than computing a Sobol set and applying Owen's scrambling. The actual implementation speed is highly dependent on the machine architecture (CPU/GPU), the environment settings, e.g., fixed or variable net sizes, and also the actual optimization tweaks in bit-level coding. For a quantitative example, we implemented Netshuffle as a class that returns a 16-point net of samples at once, parameterized by a 32-bit word of flags. Our implementation is available in the supplementary materials and may be adapted for other fixed-size nets. It delivers around $120 \mathrm{M}$ points per second, compared to around only $45 \mathrm{M}$ for unscrambled Sobol on the same machine, using PBRT's implementation of 2D Sobol [Pharr et al. 2016]. Thus, even with an $O(1)$ implementation of Owen's scrambling like Burley's [2020], Netshuffle still wins by outperforming the underlying Sobol sample generation.

The time complexity of Algorithm 4 is $O(N \log (N) \cdot T)$ per iteration, where $T$ is the time complexity of energy evaluation at a point. The first term is for iterating through the points, the second is for iterating through the stratifications, and the third is for evaluating the energy. This last term is the real bottleneck. Many blue-noise optimization algorithms consider a fixed neighborhood around each point; hence $T$ is constant. But some algorithms, e.g., BNOT [de Goes et al. 2012], use a global energy term, and therefore, are not really feasible for optimizing nets. We observed that the required number of iterations seems to be a reasonable $O(\log (N))$ or less. To give actual numbers, blue-noise optimization in a single core of a contemporary machine takes between 2 seconds ( 8 iterations) for a $(0,10,2)$-net with $1 \mathrm{~K}$ points and $6: 47$ hours (45 iterations) for a $(0,20,2)$-net with $1 \mathrm{M}$ points. Poisson-disk optimization is orders of magnitudes faster, since it only considers the immediate neighbors of a point. Thus, the throughput of Netoptimize is very competent compared to native blue-noise optimization algorithms. Notably, Netoptimize saves the effort of preparing stratified reference sets as in LDBN [Ahmed et al. 2016].

The space required to store the flags of a net is exactly one quarter of the space needed to store the points, which gives an idea about the actual dimension of dyadic nets. This cannot be compressed further. If the goal is to produce random nets, however, then a cyclic buffer of $n$ random flags may effectively store $n$ distinct nets, and may be well-suited for GPU applications.

\subsection{Integration}

In this section, we compare the integration performance of Blue Nets to various state-of-the-art LD alternatives. Following [Christensen et al. 2018], we also include Ahmed's Adaptive Regular Tiles (ART) [2017b] in this comparison. We use analytic functions similar to [Christensen et al. 2018], but with a few modifications. First, we report the variance, rather than the average error, which we find more appropriate. Secondly, we dropped the step function, since it depends on only one point and is exclusively dependent on the least significant bits beyond the $m$-bits that identify the net in our convention. Finally, we added a complex, smooth integrand by placing Gaussian kernels at 64 random points of a toroidal domain. We set the $\sigma$ of these kernels to $\sqrt{1 /(8 \cdot 24 \cdot \ln (2))}$, so that their density almost vanishes at a distance of 0.5 with floating-point precision. This should give an idea about the integration performance of the point sets on a smooth texture.

Fig. 13 shows the integration results from the different sampling sets. Overall, our Blue Nets exhibit a superior and consistent performance. The separable nature of the bi-linear and the single Gaussian integrands discriminate between dyadic and non-dyadic distributions. The sudden jump in Owen-scrambled Sobol in the bilinear integrand and its gradual drift in the Gaussian are unexpected and warrant further investigation. The behavior of the different samplers is interesting with respect to the sum-of-Gaussians integrand. It is also interesting to note that both BNLD and PMJ02BN eventually overtake Blue Nets. This implies that these distributions contain less energy near zero. In contrast, our Blue Nets maintain a wider band of low energy, as evident in Fig. 11. While this is not yet conclusive, it strongly suggests that optimizing the dyadic nets and sequences matters.

\subsection{Actual Rendering}

Rendering is arguably the most important application of sampling patterns. We recall that the sampler in a rendering pipeline does two jobs: producing sets of samples, and allocating different sets for different pixels. Our current work is confined to the first function. Our nets may readily be used with Georgiev and Fajardo's dithering strategy [2016], or adopted (with some effort) for use with the sampler model of Heitz et al. [2019]. They may be padded into higher-dimensional orthogonal arrays, as described by Jarosz et al. 


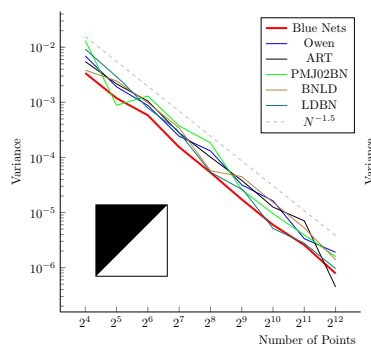

(a) Triangle

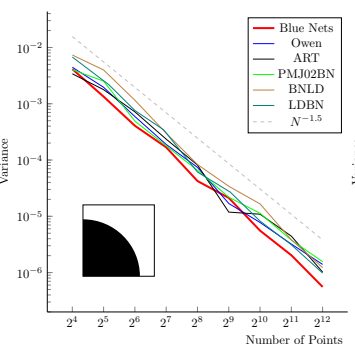

(b) Disk

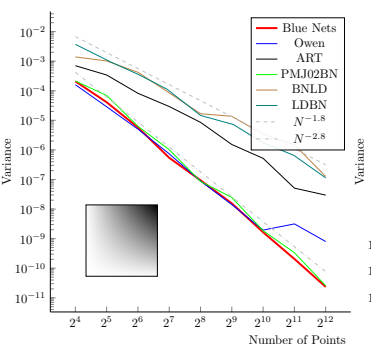

(c) Bi-linear

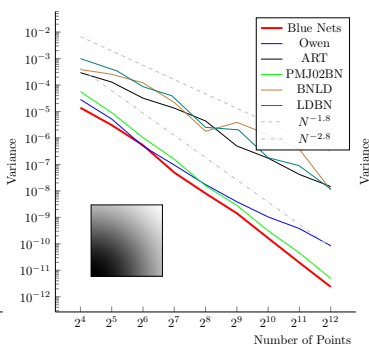

(d) Gaussian

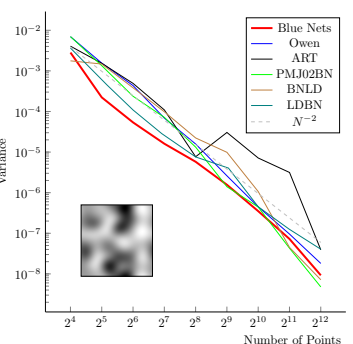

(e) Sum of Gaussians

Fig. 13. Integration variance of different sample sets using different analytic functions: (a) a triangular function that returns 1 where $y>x, 0$ otherwise, (b) a disk function that returns 1 for $x^{2}+y^{2}<2 / \pi, 0$ otherwise, (c) a bilinear function that returns $x \cdot y$, (d) a single Gaussian function that returns $\exp \left(x^{2}+y^{2}\right)$, and (e) a smooth, toroidal sum of 64 Gaussian kernels placed at random points. The integrand is shown in each plot, where a darker color indicates larger values. We averaged over 100 point sets for all samplers except PMJ02BN, where we used the 64 sets shipped with Andrew Helmer's implementation [2020]. For Owen's scrambling, we used the code in Coeurjolly and Perrier's Uni(corn|form) Tool Kit [2019]. For LDBN and BNLD, the published code generates deterministic sets, so we applied Cranley-Patterson [1976] toroidal-shifting to vary the samples. Such shifts slightly degrade the low-discrepancy quality of the point sets, but have no negative effect on the sum-of-Gaussians integrand that we designed to be toroidal.

[2019], or just used as a drop-in replacement in [Kollig and Keller 2002].

To compare the performance of the underlying sampling sets, however, we only compare our optimized nets to baseline samplers shipped with PBRT, namely the $(0,2)$-sequence, global Sobol, and global Halton. To that end, we implement a very basic sampler that uses a single blue-noise-optimized net for each point count up to 4096, applying Cranley-Patterson [1976] toroidal shifts to decorrelate the samples across pixels, and shuffling the order of the samples to decorrelate them across dimensions. For each size, we generate a large number (400) of nets by combining energy and conflict optimization, then pick the one with largest Poisson-disk radius. We treat the image plane differently. We tile the $4 \mathrm{k} / 2 \mathrm{k}$ net for an even/odd-power-of-two samples per pixel, so that the samples can be shared between adjacent pixels.

Fig. 14 shows the rendering results in two fundamentally different sampling scenarios. We encourage the reader to view the full-scale images in the supplementary materials. In Fig. 14(a), we show the application of optimized nets for anti-aliasing in image-plane sampling, using a 3-pixel Gaussian filter to demonstrate the advantage of sharing the samples between pixels. The optimized nets clearly lead the competition, both in the visual quality and the measured error, with the Sobol sampler exhibiting aliasing patterns, the $(0,2)$ sequence showing excessive noise (independent pixel sampling), and the Halton sampler showing both.

The second scene, in Fig. 14(b), is a typical Monte Carlo integration scenario, simulating a depth-of-field effect by sampling the camera lens. Again, Sobol is aliased, (0,2)-sequence is noisy, Halton shows both aliasing and noise, while our optimized nets deliver a good balance between noise and aliasing, as well as the smallest error. Even though the renderings of Sobol and Halton global samplers have larger errors in terms of RMSE, they still arguably look more eye-pleasing than our Blue Nets. This is related to the way the samples are distributed among the pixels. Indeed, we have already seen in Section 6.4 that Blue Nets are advantageous for integration. However, as of this writing, we have not yet implemented a state-of-the-art integration into the rendering system. Hence, global samplers have an inherent advantage due to their error-diffusion capability; cf. [Ahmed and Wonka 2020]. Analyzing this requires additional future work. The conclusion we draw here is that studying the manifestation of the Monte Carlo sampling error, not only its amount, must be emphasized more [Ahmed and Wonka 2020; Georgiev and Fajardo 2016; Heitz and Belcour 2019; Heitz et al. 2019].

\section{CONCLUSION}

In this paper, we made several contributions to the construction and analysis of dyadic nets (DNs). We proposed a hierarchical construction algorithm that spans all possible DNs. From this construction algorithm, we derived a parameterization of the complete space of DNs and enumerated all distinct nets. We identified the available degrees of freedom for transforming between nets, and explained how to apply these transformations to design an efficient algorithm for generating optimized nets obeying a user-specified criterion. As the most important example, we optimized for nets that have a bluenoise frequency spectrum. In addition, we proposed an algorithm for constructing a DN to match a target distribution, and gave an explanation of why the lack of degrees of freedom makes upsampling DNs and constructing optimized sequences very difficult.

One of the most important conclusions of our work is that bluenoise and low-discrepancy are quite compatible. Indeed, we managed to combine the best known discrepancy bounds with the finestquality blue-noise properties. Our basic rendering test strongly suggests that blue-noise optimization of dyadic nets is worthwhile. The application and performance of blue-noise sets in integration is not sufficiently studied yet. Indeed, theoretical analyses, like Durand's technical report [2011] and some follow-up papers [Öztireli 2016; Pilleboue et al. 2015; Ramamoorthi et al. 2012; Subr and Kautz 2013] suggest that blue noise is advantageous for integration, yet there is no sufficient empirical evidence. The results in Section 6.4 are enlightening, suggesting that, at least for some functions, combined low-discrepancy and blue-noise sample sets, e.g., Blue Nets, PMJ02BN, or BNLD, are superior to sets with only one property, e.g., Owen-scrambled Sobol or ART. It is too early to draw conclusions 


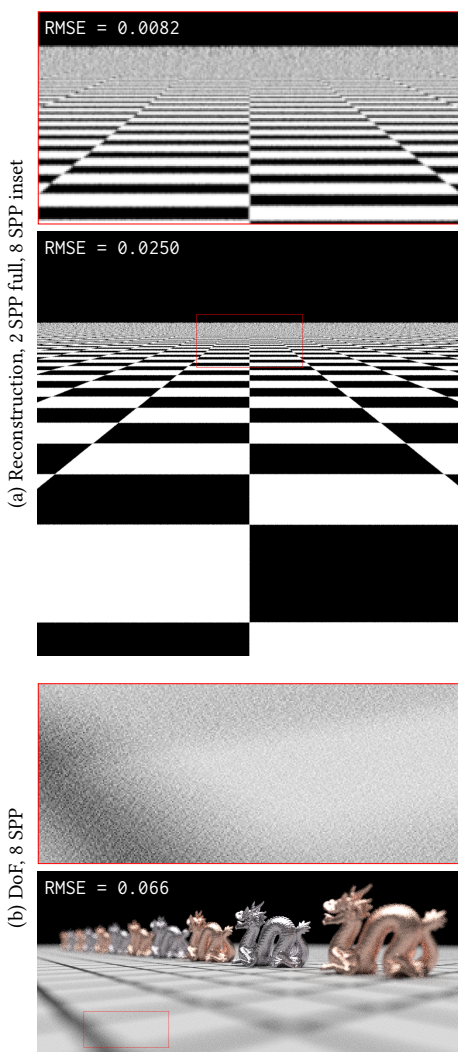

(A) Halton

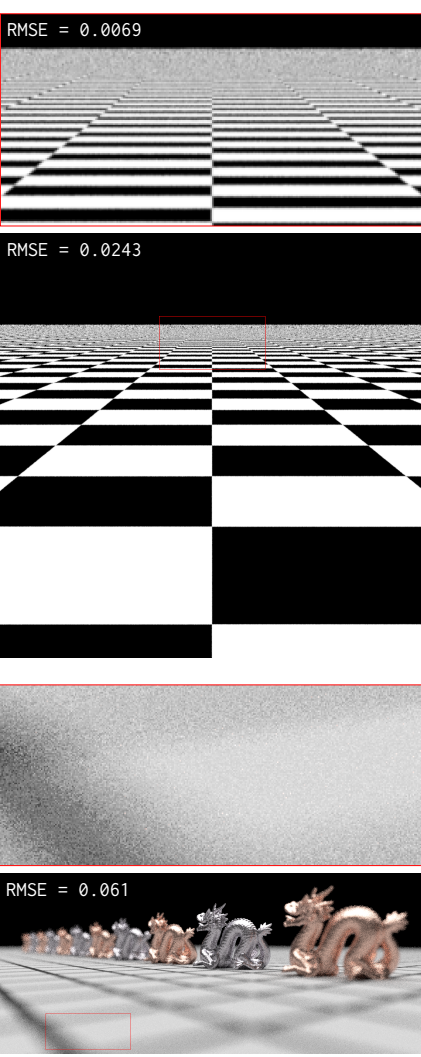

(B) $(0,2)$-Sequence

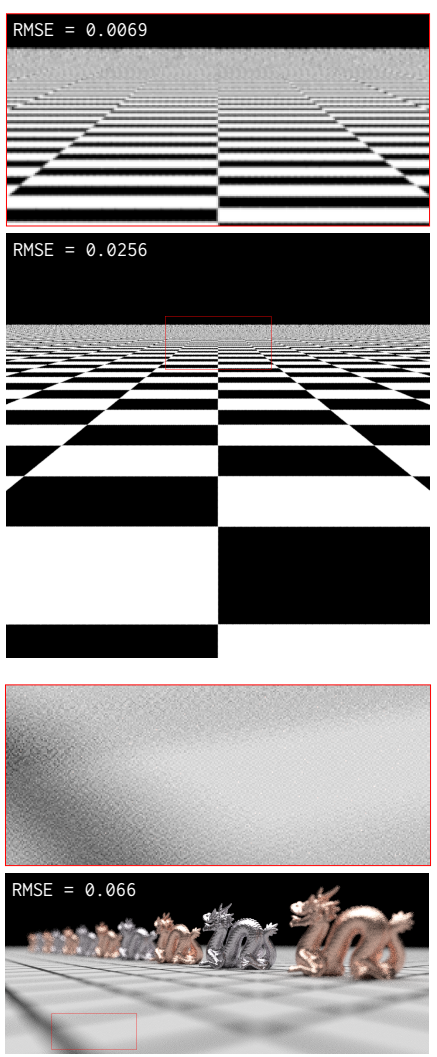

(C) Sobol
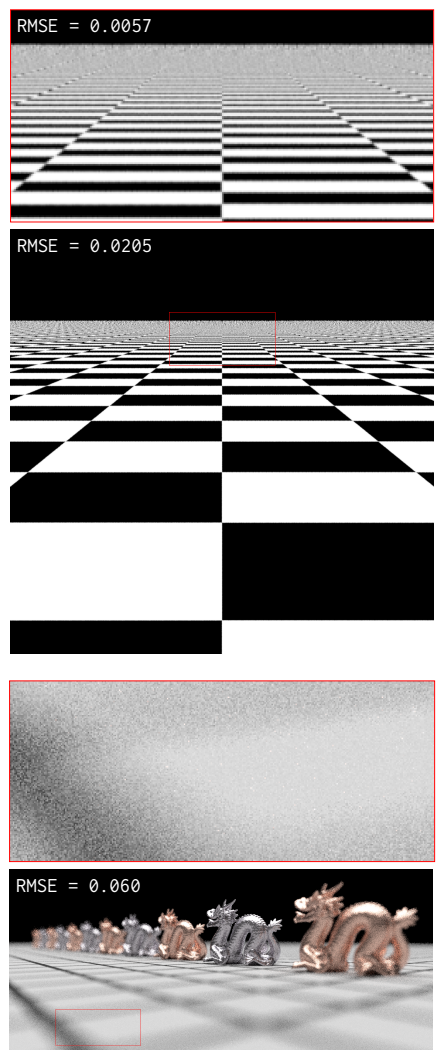

(D) Blue Nets (Ours)

Fig. 14. Comparison of renderings using Blue Nets and state-of-the-art samplers in scenes emphasizing two different sampling applications: (a) reconstruction in the image plane, and (b) integration targeting the lens.

though, and we hope these insights encourage future research on this topic. We hope the tools and models contributed in this paper will facilitate such research.

We believe that our core construction algorithm provides the essential missing ingredient for understanding the generation and design space of dyadic nets. Therefore, it opens the door to many new research questions and directions that have not been explored in this initial work. First, we did not explore complementary questions that might arise in applications. We can identify three challenges in this context. Point sets can be extended to higher dimensions by padding, they can be extended to a larger spatial extent by tiling, and they can be assigned to pixels, e.g., Ahmed and Wonka [2020] The configuration of each of these steps needs to be coordinated, and their interplay should be analyzed. We leave a study of these issues to future work. Second, besides the extension to higher dimensions using padding, one could wish for a direct extension, e.g., by analyzing the design space of 3D, 4D, or higher dimensional nets We do believe that this will also require a significant separate effort in the future. Third, we are interested in studying how different parameterizations of DNs can facilitate a combination with machine learning algorithms. Is it possible to design a network architecture and training strategy that can learn to construct DNs and then adapt them to given design criteria? It would be interesting to see a solution for style transfer to point sets, without destroying the digital net property. Finally, we would like to expand our work to more directly tackle sequence construction under arbitrary user-provided objective functions. Our work indicates that this will be challenging due to the greatly reduced design space, but we consider this to be a worthwhile challenge to take on.

\section{ACKNOWLEDGMENTS}

Thanks to the anonymous reviewers for the valuable comments. Thanks to Mohanad Ahmed for his insightful discussions.

\section{REFERENCES}

A. G. M. Ahmed, J. Guo, D. M. Yan, J. Y. Franceschia, X. Zhang, and O. Deussen. 2017a. A Simple Push-Pull Algorithm for Blue-Noise Sampling. IEEE Transactions on Visualization and Computer Graphics 23, 12 (Dec. 2017), 2496-2508. https: //doi.org/10.1109/TVCG.2016.2641963

Abdalla G. M. Ahmed, Till Niese, Hui Huang, and Oliver Deussen. 2017b. An Adaptive Point Sampler on a Regular Lattice. ACM Trans. Graph. 36, 4, Article 138 (July 2017), 13 pages. https://doi.org/10.1145/3072959.3073588

Abdalla G. M. Ahmed, Hélène Perrier, David Coeurjolly, Victor Ostromoukhov, Jianwei Guo, Dong-Ming Yan, Hui Huang, and Oliver Deussen. 2016. Low-Discrepancy Blue-Noise Sampling. ACM Trans. Graph. 35, 6, Article 247 (Nov. 2016), 13 pages. https://doi.org/10.1145/2980179.2980218

Abdalla G. M. Ahmed and Peter Wonka. 2020. Screen-Space Blue-Noise Diffusion of Monte Carlo Sampling Error via Hierarchical Ordering of Pixels. ACM Trans. Graph. 
39, 6, Article 244 (Nov. 2020), 15 pages. https://doi.org/10.1145/3414685.3417881

I.A. Antonov and V.M. Saleev. 1979. An Economic Method of Computing LP-sequences. U. S. S. R. Comput. Math. and Math. Phys. 19, 1 (1979), 252-256. https://doi.org/10. 1016/0041-5553(79)90085-5

Michael Balzer, Thomas Schlömer, and Oliver Deussen. 2009. Capacity-Constrained Point Distributions: A Variant of Lloyd's Method. ACM Trans. Graph. 28, 3, Article 86 (July 2009), 8 pages. https://doi.org/10.1145/1531326.1531392

R. Bridson. 2007. Fast Poisson-Disk Sampling in Arbitrary Dimensions. In ACM SIGGRAPH 2007 Sketches.

Brent Burley. 2020. Practical Hash-Based Owen Scrambling. Fournal of Computer Graphics Techniques (FCGT) 10, 4 (29 December 2020), 1-20. http://jcgt.org/published/ 0009/04/01/

Zhonggui Chen, Zhan Yuan, Yi-King Choi, Ligang Liu, and Wenping Wang. 2012. Variational Blue Noise Sampling. IEEE Transactions on Visualization and Computer Graphics 18, 10 (Oct. 2012), 1784-1796. https://doi.org/10.1109/TVCG.2012.94

Per Christensen, Andrew Kensler, and Charlie Kilpatrick. 2018. Progressive MultiJittered Sample Sequences. In Computer Graphics Forum, Vol. 37. Wiley Online Library, 21-33.

David Coeurjolly and Hélène Perrier. 2019. Uni(corn|form) Tool Kit. https://utkteam.github.io/utk/, as of 2021-01-27.

Robert L. Cook. 1986. Stochastic Sampling in Computer Graphics. ACM Trans. Graph. 5, 1 (Jan. 1986), 51-72. https://doi.org/10.1145/7529.8927

Roy Cranley and Thomas NL Patterson. 1976. Randomization of Number-Theoretic Methods for Multiple Integration. SIAM 7. Numer. Anal. 13, 6 (1976), 904-914.

Josef Dick and Friedrich Pillichshammer. 2010. Digital Nets and Sequences: Discrepancy Theory and Quasi-Monte Carlo Integration. Cambridge University Press. https: //doi.org/10.1017/CBO9780511761188

Mark A. Z. Dippé and Erling Henry Wold. 1985. Antialiasing through Stochastic Sampling. In Proceedings of the 12th Annual Conference on Computer Graphics and Interactive Techniques (SIGGRAPH '85). ACM, 69-78. https://doi.org/10.1145/325334. 325182

Daniel Dunbar and Greg Humphreys. 2006. A Spatial Data Structure for Fast PoissonDisk Sample Generation. ACM Trans. Graph. 25, 3 (July 2006), 503-508. https: //doi.org/10.1145/1141911.1141915

Fredo Durand. 2011. A Frequency Analysis of Monte Carlo and Other Numerical Integration Schemes. MIT CSAIL Tech. Rep. TR-2011-052 (2011)

Mohamed S. Ebeida, Muhammad A. Awad, Xiaoyin Ge, Ahmed H. Mahmoud, Scott A. Mitchell, Patrick M. Knupp, and Li-Yi Wei. 2014. Improving Spatial Coverage while Preserving the Blue Noise of Point Sets. Computer-Aided Design 46, Supplement C (2014), 25-36. https://doi.org/10.1016/j.cad.2013.08.015 2013 SIAM Conference on Geometric and Physical Modeling.

Mohamed S. Ebeida, Andrew A. Davidson, Anjul Patney, Patrick M. Knupp, Scott A Mitchell, and John D. Owens. 2011. Efficient Maximal Poisson-Disk Sampling. ACM Trans. Graph. 30, 4, Article 49 (July 2011), 12 pages. https://doi.org/10.1145/2010324. 1964944

Mohamed S. Ebeida, Scott A. Mitchell, Anjul Patney, Andrew A. Davidson, and John D. Owens. 2012. A Simple Algorithm for Maximal Poisson-Disk Sampling in High Dimensions. Comp. Graph. Forum 31, 2pt4 (May 2012), 785-794. https://doi.org/10. 1111/j.1467-8659.2012.03059.x

Y. Eldar, M. Lindenbaum, M. Porat, and Y. Y. Zeevi. 1997. The Farthest-Point Strategy for Progressive Image Sampling. Trans. Img. Proc. 6, 9 (Sept. 1997), 1305-1315. https://doi.org/10.1109/83.623193

Raanan Fattal. 2011. Blue-Noise Point Sampling Using Kernel Density Model. In ACM SIGGRAPH 2011 Papers (Vancouver, British Columbia, Canada) (SIGGRAPH '11). ACM, Article 48, 12 pages. https://doi.org/10.1145/1964921.1964943

Henri Faure and Shu Tezuka. 2002. Another Random Scrambling of Digital $(\mathrm{t}, \mathrm{s})$ Sequences. In Monte Carlo and Quasi-Monte Carlo Methods 2000, Kai-Tai Fang, Harald Niederreiter, and Fred J. Hickernell (Eds.). Springer, 242-256.

Manuel N. Gamito and Steve C. Maddock. 2009. Accurate Multidimensional PoissonDisk Sampling. ACM Trans. Graph. 29, 1, Article 8 (Dec. 2009), 19 pages. https //doi.org/10.1145/1640443.1640451

Iliyan Georgiev and Marcos Fajardo. 2016. Blue-Noise Dithered Sampling. In $A C M$ SIGGRAPH 2016 Talks. 1-1.

Fernando de Goes, Katherine Breeden, Victor Ostromoukhov, and Mathieu Desbrun. 2012. Blue Noise through Optimal Transport. ACM Trans. Graph. 31, 6, Article 171 (Nov. 2012), 11 pages. https://doi.org/10.1145/2366145.2366190

Leonhard Grünschloß, Johannes Hanika, Ronnie Schwede, and Alexander Keller. 2008 $(\mathrm{t}, \mathrm{m}, \mathrm{s})$-Nets and Maximized Minimum Distance. In Monte Carlo and Quasi-Monte Carlo Methods 2006, Alexander Keller, Stefan Heinrich, and Harald Niederreiter (Eds.). Springer, 397-412.

Leonhard Grünschloß and Alexander Keller. 2009. (t, m, s)-Nets and Maximized Minimum Distance, Part II. In Monte Carlo and Quasi-Monte Carlo Methods 2008. Springer, 395-409.

Leonhard Grünschloß, Matthias Raab, and Alexander Keller. 2012. Enumerating Quasi-Monte Carlo Point Sequences in Elementary Intervals. In Monte Carlo and Quasi-Monte Carlo Methods 2010, Leszek Plaskota and Henryk Woźniakowski (Eds.).
Springer, 399-408.

Kenneth M. Hanson. 2003. Quasi-Monte Carlo: Halftoning in High Dimensions? In Computational Imaging, Charles A. Bouman and Robert L. Stevenson (Eds.), Vol. 5016. International Society for Optics and Photonics, SPIE, $161-172$. https: //doi.org/10.1117/12.484808

Kenneth M Hanson. 2005. Halftoning and Quasi-Monte Carlo. Los Alamos National Library (2005), 430-442.

Daniel Heck, Thomas Schlömer, and Oliver Deussen. 2013. Blue Noise Sampling with Controlled Aliasing. ACM Trans. Graph. 32, 3, Article 25 (July 2013), 12 pages. https://doi.org/10.1145/2487228.2487233

Eric Heitz and Laurent Belcour. 2019. Distributing Monte Carlo Errors as a Blue Noise in Screen Space by Permuting Pixel Seeds Between Frames. In Computer Graphics Forum, Vol. 38. Wiley Online Library, 149-158.

Eric Heitz, Laurent Belcour, V. Ostromoukhov, David Coeurjolly, and Jean-Claude Iehl. 2019. A Low-Discrepancy Sampler That Distributes Monte Carlo Errors as a Blue Noise in Screen Space. In ACM SIGGRAPH 2019 Talks (Los Angeles, California) (SIGGRAPH '19). Association for Computing Machinery, Article 68, 2 pages. https: //doi.org/10.1145/3306307.3328191

Andrew Helmer. 2020. A C++ implementation of Progressive Multi-Jittered Sample Sequences. https://github.com/Andrew-Helmer/pmj-cpp/tree/master/sample sequences, as of 2021-04-23.

Wojciech Jarosz, Afnan Enayet, Andrew Kensler, Charlie Kilpatrick, and Per Christensen 2019. Orthogonal Array Sampling for Monte Carlo Rendering. In Computer Graphics Forum, Vol. 38. Wiley Online Library, 135-147.

Min Jiang, Yahan Zhou, Rui Wang, Richard Southern, and Jian Jun Zhang. 2015. Blue Noise Sampling Using an SPH-Based Method. ACM Trans. Graph. 34, 6, Article 211 (2015), 11 pages. https://doi.org/10.1145/2816795.2818102

Thouis R Jones. 2006. Efficient Generation of Poisson-Disk Sampling Patterns. Fournal of graphics, gpu, and game tools 11, 2 (2006), 27-36.

Alexander Keller. 2006. Myths of Computer Graphics. In Monte Carlo and Quasi-Monte Carlo Methods 2004. Springer, 217-243.

Alexander Keller. 2013. Quasi-Monte Carlo Image Synthesis in a Nutshell. In Monte Carlo and Quasi-Monte Carlo Methods 2012, Josef Dick, Frances Y. Kuo, Gareth W. Peters, and Ian H. Sloan (Eds.). Springer, 213-249.

Alexander Keller, Iliyan Georgiev, Abdalla Ahmed, Per Christensen, and Matt Pharr 2019. My Favorite Samples. In ACM SIGGRAPH 2019 Courses (Los Angeles, California) (SIGGRAPH '19). Association for Computing Machinery, Article 15, 271 pages. https: //doi.org/10.1145/3305366.3329901

Andrew Kensler. 2013. Correlated Multi-Jittered Sampling. Pixar Technical Memo 13-01 7 (2013), 86-112.

Thomas Kollig and Alexander Keller. 2002. Efficient Multidimensional Sampling. In Computer Graphics Forum, Vol. 21. 557-563.

Lauwerens Kuipers and Harald Niederreiter. 1974. Uniform Distribution of Sequences John Wiley \& Sons. http://opac.inria.fr/record=b1083239 A Wiley-Interscience publication.

Ares Lagae and Philip Dutré. 2008. A Comparison of Methods for Generating PoissonDisk Distributions. In Computer Graphics Forum, Vol. 27. Wiley Online Library, 114-129.

G Larcher and F Pillichshammer. 2003. Sums of Distances to the Nearest Integer and the Discrepancy of Digital Nets. Acta Arithmetica 106 (2003), 379-408.

Michael McCool and Eugene Fiume. 1992. Hierarchical Poisson-Disk Sampling Distributions. In Proceedings of the Conference on Graphics Interface '92 (Vancouver, British Columbia, Canada). Morgan Kaufmann Publishers Inc., San Francisco, CA, USA, 94-105. http://dl.acm.org/citation.cfm?id=155294.155306

Don P. Mitchell. 1991. Spectrally Optimal Sampling for Distribution Ray Tracing. SIGGRAPH Comput. Graph. 25, 4 (July 1991), 157-164. https://doi.org/10.1145/ 127719.122736

Don P Mitchell. 1992. Ray Tracing and Irregularities of Distribution. In Third Eurographics Workshop on Rendering. 61-69.

Scott A. Mitchell, Mohamed S. Ebeida, Muhammad A. Awad, Chonhyon Park, Anjul Patney, Ahmad A. Rushdi, Laura P. Swiler, Dinesh Manocha, and Li-Yi Wei. 2018 Spoke-Darts for High-Dimensional Blue-Noise Sampling. ACM Trans. Graph. 37, 2 , Article 22 (May 2018), 20 pages. https://doi.org/10.1145/3194657

Harald Niederreiter. 1987. Point Sets and Sequences with Small Discrepancy. Monatshefte für Mathematik 104, 4 (1987), 273-337.

Harald Niederreiter. 1988. Low-Discrepancy and Low-Dispersion Sequences. fournal of Number Theory 30, 1 (1988), 51 - 70. https://doi.org/10.1016/0022-314X(88)90025-X

Harald Niederreiter. 1992. Random Number Generation and Quasi-Monte Carlo Methods. SIAM

Harald Niederreiter. 2005. Constructions of $(\mathrm{t}, \mathrm{m}, \mathrm{s})$-Nets and $(\mathrm{t}, \mathrm{s})$-Sequences. Finite Fields and Their Applications 11, 3 (2005), 578-600. https://doi.org/10.1016/j.ffa.2005.01.001 Ten Year Anniversary Edition!

Art B. Owen. 1995. Randomly Permuted (t,m,s)-Nets and (t, s)-Sequences. In Monte Carlo and Quasi-Monte Carlo Methods in Scientific Computing, Harald Niederreiter and Peter Jau-Shyong Shiue (Eds.). Springer, 299-317. 
Art B. Owen. 1998. Scrambling Sobol'and Niederreiter-Xing Points. Fournal of Complexity 14, 4 (1998), 466-489.

Art B. Owen. 2003. Variance with Alternative Scramblings of Digital Nets. ACM Trans Model. Comput. Simul. 13, 4 (Oct. 2003), 363-378. https://doi.org/10.1145/945511. 945518

A. Cengiz Öztireli. 2016. Integration with Stochastic Point Processes. ACM Trans. Graph. 35, 5, Article 160 (Aug. 2016), 16 pages. https://doi.org/10.1145/2932186

A. Cengiz Öztireli. 2020. A Comprehensive Theory and Variational Framework for Anti-aliasing Sampling Patterns. Computer Graphics Forum 39, 4 (2020), 133-148. https://doi.org/10.1111/cgf.14059 arXiv:https://onlinelibrary.wiley.com/doi/pdf/10.1111/cgf.14059

A. Cengiz Öztireli, Marc Alexa, and Markus Gross. 2010. Spectral Sampling of Manifolds. ACM Transactions on Graphics (TOG) 29, 6 (2010), 1-8.

A. Cengiz Öztireli and Markus Gross. 2012. Analysis and Synthesis of Point Distributions Based on Pair Correlation. ACM Trans. Graph. 31, 6, Article 170 (Nov. 2012), 10 pages. https://doi.org/10.1145/2366145.2366189

Hélène Perrier, David Coeurjolly, Feng Xie, Matt Pharr, Pat Hanrahan, and Victor Ostromoukhov. 2018. Sequences with Low-Discrepancy Blue-Noise 2-D Projections. Computer Graphics Forum (Proceedings of Eurographics) 37, 2 (2018), 339-353.

Matt Pharr. 2019. Efficient Generation of Points that Satisfy Two-Dimensional Elementary Intervals. Fournal of Computer Graphics Techniques (FCGT) 8, 1 (27 February 2019), 56-68. http://jcgt.org/published/0008/01/04/

Matt Pharr and Greg Humphreys. 2010. Physically-Based Rendering: from Theory to Implementation (2nd ed.). Morgan Kaufmann Publishers Inc., San Francisco, CA USA.

Matt Pharr, Wenzel Jakob, and Greg Humphreys. 2016. Physically Based Rendering: From Theory to Implementation (3rd ed.). Morgan Kaufmann Publishers Inc., San Francisco, CA, USA

Adrien Pilleboue, Gurprit Singh, David Coeurjolly, Michael Kazhdan, and Victor Ostromoukhov. 2015. Variance Analysis for Monte Carlo Integration. ACM Trans. Graph. 34, 4, Article 124 (July 2015), 14 pages. https://doi.org/10.1145/2766930

Ravi Ramamoorthi, John Anderson, Mark Meyer, and Derek Nowrouzezahrai. 2012. A Theory of Monte Carlo Visibility Sampling. ACM Trans. Graph. 31, 5, Article 121 (2012), 16 pages. https://doi.org/10.1145/2231816.2231819

Bernhard Reinert, Tobias Ritschel, Hans-Peter Seidel, and Iliyan Georgiev. 2016. Projective Blue-Noise Sampling. Computer Graphics Forum 35, 1 (2016), 285-295. https://doi.org/10.1111/cgf.12725

Thomas Schlömer, Daniel Heck, and Oliver Deussen. 2011. Farthest-Point Optimized Point Sets with Maximized Minimum Distance. In Proceedings of the ACM SIGGRAPH Symposium on High Performance Graphics (Vancouver, British Columbia, Canada) (HPG '11). ACM, 135-142. https://doi.org/10.1145/2018323.2018345

Thomas Schlömer and Oliver Deussen. 2011. Accurate Spectral Analysis of Two-Dimensional Point Sets. Fournal of Graphics, GPU, and Game Tools 15, 3 (2011), 152-160. https://doi.org/10.1080/2151237X.2011.609773 arXiv:http://dx.doi.org/10.1080/2151237X.2011.609773

Claude E Shannon. 1948. A Mathematical Theory of Communication. The Bell System Technical fournal 27, 3 (1948), 379-423.

Peter Shirley. 1991. Discrepancy as a Quality Measure for Sample Distributions. In Proc Eurographics '91, Vol. 91. 183-194.

Il'ya Meerovich Sobol'. 1967. On the Distribution of Points in a Cube and the Approximate Evaluation of Integrals. Zhurnal Vychislitel'noi Matematiki i Matematicheskoi Fiziki 7, 4 (1967), 784-802.

Sergej Stoppel and Stefan Bruckner. 2019. LinesLab: A Flexible Low-Cost Approach for the Generation of Physical Monochrome Art. In Computer Graphics Forum, Vol. 38 Wiley Online Library, 110-124.

Kartic Subr and Jan Kautz. 2013. Fourier Analysis of Stochastic Sampling Strategies for Assessing Bias and Variance in Integration. ACM Trans. Graph. 32, 4, Article 128 (2013), 12 pages. https://doi.org/10.1145/2461912.2462013

Robert Ulichney. 1987. Digital Halftoning. MIT Press, Cambridge, MA, USA.

R.A. Ulichney. 1988. Dithering with Blue Noise. Proc. IEEE 76, 1 (Jan 1988), 56-79. https://doi.org/10.1109/5.3288

Robert A Ulichney. 1993. Void-and-Cluster Method for Dither Array Generation. In IS\&T/SPIE's Symposium on Electronic Imaging: Science and Technology. International Society for Optics and Photonics, 332-343.

J.G. van der Corput. 1935. Verteilungsfunktionen. Proceedings of the Nederlandse Akademie van Wetenschappen 38 (1935), 813-821.

Li-Yi Wei. 2010. Multi-Class Blue-Noise Sampling. ACM Trans. Graph. 29, 4, Article 79 (July 2010), 8 pages. https://doi.org/10.1145/1778765.1778816

SK Zaremba. 1968. The Mathematical Basis of Monte Carlo and Quasi-Monte Carlo Methods. SIAM review 10, 3 (1968), 303-314.

Yahan Zhou, Haibin Huang, Li-Yi Wei, and Rui Wang. 2012. Point Sampling with General Noise Spectrum. ACM Trans. Graph. 31, 4, Article 76 (July 2012), 11 pages. https://doi.org/10.1145/2185520.2185572

\section{A ALGEBRAIC DERIVATION}

In this appendix, we derive an expression for $g_{i, j}(\mathbf{x})$ in Eq. (24). Each point can be broken down axis-wise and aggregated back later, so looking only at the $x$-axis, for example,

$$
\begin{aligned}
& g_{i, j}(x)=\exp \left(-\frac{\left(x-x_{i}\right)^{2}+\left(x-x_{j}\right)^{2}}{2 \sigma^{2}}\right) \\
& =\exp \left(-\frac{x^{2}-2 x x_{i}+x_{i}^{2}+x^{2}-2 x x_{j}+x_{j}^{2}}{2 \sigma^{2}}\right) \\
& =\exp \left(-\frac{2 x^{2}-2 x\left(x_{i}+x_{j}\right)+x_{i}^{2}+x_{j}^{2}}{2 \sigma^{2}}\right) \\
& =\exp \left(-\frac{x^{2}-2 x \frac{x_{i}+x_{j}}{2}+\left(\frac{x_{i}+x_{j}}{2}\right)^{2}-\left(\frac{x_{i}+x_{j}}{2}\right)^{2}+\frac{x_{i}^{2}+x_{j}^{2}}{2}}{\sigma^{2}}\right) \\
& =\exp \left(-\frac{\left(x-\frac{x_{i}+x_{j}}{2}\right)^{2}+\frac{x_{i}^{2}+x_{j}^{2}}{2}-\frac{x_{i}^{2}+2 x_{i} x_{j}+x_{j}^{2}}{4}}{\sigma^{2}}\right) \\
& =\exp \left(-\frac{\left(x-\frac{x_{i}+x_{j}}{2}\right)^{2}+\frac{x_{i}^{2}-2 x_{i} x_{j}+x_{j}^{2}}{4}}{\sigma^{2}}\right) \\
& =\exp \left(-\frac{\left(x-\frac{x_{i}+x_{j}}{2}\right)^{2}+\frac{\left(x_{i}-x_{j}\right)^{2}}{4}}{\sigma^{2}}\right) \\
& =\exp \left(-\frac{\left(x_{i}-x_{j}\right)^{2}}{4 \sigma^{2}}\right) \exp \left(-\frac{\left(x-\frac{x_{i}+x_{j}}{2}\right)^{2}}{\sigma^{2}}\right) \text {. }
\end{aligned}
$$

Hence,

$$
g_{i, j}(\mathbf{x})=\exp \left(-\frac{\left\|\mathbf{x}_{i}-\mathbf{x}_{j}\right\|^{2}}{4 \sigma^{2}}\right) \exp \left(-\frac{\left\|\mathbf{x}-\frac{\mathbf{x}_{i}+\mathbf{x}_{j}}{2}\right\|^{2}}{\sigma^{2}}\right) .
$$

